ALEA, Lat. Am. J. Probab. Math. Stat. 15, 509-547 (2018)

DOI: 10.30757/ALEA.v15-21

\title{
Fluctuation exponents for stationary exactly solvable lattice polymer models via a Mellin transform framework
}

\author{
Hans Chaumont and Christian Noack \\ University of Wisconsin Madison \\ Van Vleck Hall, \\ 480 Lincoln Drive \\ Madison, WI, United States. \\ E-mail address: chaumont@wisc.edu \\ URL: http://www.math.wisc.edu/ chaumont \\ University of Wisconsin Madison \\ Van Vleck Hall, \\ 480 Lincoln Drive \\ Madison, WI, United States. \\ E-mail address: cnoack@wisc.edu \\ URL: http://www.math.wisc.edu/ cnoack
}

\begin{abstract}
We develop a Mellin transform framework which allows us to simultaneously analyze the four known exactly solvable $1+1$ dimensional lattice polymer models: the log-gamma, strict-weak, beta, and inverse-beta models. Using this framework we prove the conjectured fluctuation exponents of the free energy and the polymer path for the stationary point-to-point versions of these four models. The fluctuation exponent for the polymer path was previously unproved for the strict-weak, beta, and inverse-beta models. An independent and concurrent work by Balázs et al. (2018) also gives the path fluctuation result for the beta model.
\end{abstract}

\section{Introduction}

The directed polymer in a random environment was first introduced by Huse and Henley (1985) to model the interaction between a long chain of molecules and random impurities. This was later reformulated by Imbrie and Spencer (1988) as a random walk in a random environment. See the recent lectures notes by Comets (2017) for additional historical background and a survey of techniques used to study directed polymers. In the $1+1$ dimensional case, a large class of

Received by the editors December 12th, 2017; accepted March 23th, 2018.

2010 Mathematics Subject Classification. 60K35; 60K37; 82B20; 82B23; 82D60.

Key words and phrases. Directed polymer, exactly solvable models, integrable models, Burke's theorem, partition function, fluctuation exponent, scaling exponent. 
polymer models are expected to lie in the KPZ universality class. For this class, the polymer path and free energy fluctuation exponents are conjectured to be $2 / 3$ and $1 / 3$, respectively, and the probability distribution of the rescaled free energy is conjectured to converge to the Tracy-Widom GUE distribution.

There are a few $1+1$ dimensional polymer models for which these results have been proved. Balázs et al. (2011), prove the fluctuation exponents for a Hopf-Cole solution to the KPZ equation with Brownian initial condition. This solution can be interpreted as the free energy of a stationary continuum directed polymer. Amir et al. (2011) study the Hopf-Cole solution to the KPZ equation with narrow-wedge initial condition and prove Tracy-Widom limit distribution for large time. For the semi-discrete Brownian directed polymer introduced by O'Connell and Yor (2001), the fluctuation exponents are proved by Seppäläinen and Valkó (2010), and the limit distribution is proved by Borodin and Corwin (2014) and Borodin et al. (2014).

In the setting of lattice directed polymers, there are four models for which results about the scaling exponents or limit distributions are known. The log-gamma directed polymer was introduced by Seppäläinen (2012), where the fluctuation exponents were proved. The limit distribution result was proved by Borodin et al. (2013). The strict-weak polymer model was simultaneously introduced by Corwin et al. (2015) and O'Connell and Ortmann (2015). Its limit distribution was proved through different methods in these two papers. The beta directed polymer was introduced by Barraquand and Corwin (2017), where its limit distribution was also calculated. The fourth model is the inverse-beta model, introduced by Thiery and Le Doussal (2015), in which they conjecture a formula for the Laplace transform of the polymer partition function and, contingent on this conjecture, show TracyWidom limit distribution for the rescaled free energy.

In this paper we provide a Mellin transform framework with which we are able to treat these four lattice polymer models simultaneously and prove the fluctuation exponents of the free energy and the polymer path for their stationary versions. While for the log-gamma model these results were previously shown by Seppäläinen (2012), for the strict-weak, beta, and inverse-beta models, the path fluctuation results are new. An independent and concurrent work by Balázs et al. (2018) also gives the path fluctuation result for the beta model.

Our methods rely upon a Burke-type stationarity property that each of these models possesses. This stationarity, along with a coupling argument, are used to prove a variance formula which is then amenable to analysis. This method was first used by Cator and Groeneboom (2006) to prove the order of the variance of the length of the longest weakly North-East path in Hammersley's process with sources and sinks. Balázs et al. (2006) adapt this method to prove the order of the fluctuations of the passage time and the fluctuations of the maximal path for last passage percolation with exponential weights. Seppäläinen (2012) used this method to prove the order of the fluctuation of the free energy and the polymer path fluctuations for the point-to-point log-gamma model with stationary boundary conditions, and upper bounds on the fluctuation exponents for the non-stationary point-to-point and point-to-line models. Seppäläinen and Valkó (2010) prove the scaling exponents for the O'Connell-Yor polymer, and Moreno Flores et al. (2014) extend the result to the intermediate disorder regime. Our paper closely follows the methods in Seppäläinen (2012); the Mellin transform framework provides a unified way to apply these methods to the four models. 
In our related paper Chaumont and Noack (2017) we prove that in the setting of $1+1$ dimensional lattice directed polymers, the only four models possessing the Burke-type stationarity property are the log-gamma, strict-weak, beta, and inversebeta models.

Notation: $\mathbb{N}=\{1,2, \ldots\}, \mathbb{Z}_{+}=\{0,1, \ldots\}$, and $\mathbb{R}$ denotes the real numbers. Let $\lfloor x\rfloor$ denote the greatest integer less than or equal to $x$. Let $\vee$ and $\wedge$ denote maximum and minimum, respectively. Given a real valued function $f$, let $\operatorname{supp}(f)=$ $\{x: f(x) \neq 0\}$ denote the support of the function $f$ (note that we do not insist on taking the closure of this set). Given a random variable $X$ with finite expectation, we let $\bar{X}=X-\mathbb{E}[X]$. For $A \subset \mathbb{R}$ write $-A=\{-a: a \in A\}$ and $A^{-1}=\left\{a^{-1}: a \in A\right\}$ assuming that $0 \notin A$. The symbol $\otimes$ is used to denote (independent) product distribution.

1.1. The polymer model. On each edge $e$ of the $\mathbb{Z}_{+}^{2}$ lattice we place a positive random weight. The superscripts 1 and 2 will be used to denote horizontal and vertical edge weights, respectively. For $z \in \mathbb{N}^{2}$, let $Y_{z}^{1}$ and $Y_{z}^{2}$ denote the horizontal and vertical incoming edge weights. We assume that the collection of pairs $\left\{\left(Y_{z}^{1}, Y_{z}^{2}\right)\right\}_{z \in \mathbb{N}^{2}}$ is independent and identically distributed with common distribution $\left(Y^{1}, Y^{2}\right)$, but do not insist that $Y_{z}^{1}$ is independent of $Y_{z}^{2}$. Call this collection the bulk weights. For $x \in \mathbb{N} \times\{0\}$, let $R_{x}^{1}$ denote the horizontal incoming edge weight, and for $y \in\{0\} \times \mathbb{N}$, let $R_{y}^{2}$ denote the vertical incoming edge weight. We assume the collections $\left\{R_{x}^{1}\right\}_{x \in \mathbb{N} \times\{0\}}$ and $\left\{R_{y}^{2}\right\}_{y \in\{0\} \times \mathbb{N}}$ are independent and identically distributed with common distributions $R^{1}$ and $R^{2}$, and refer to them as the horizontal and vertical boundary weights, respectively. We further assume that the horizontal boundary weights, the vertical boundary weights, and the bulk weights are independent of each other. This assignment of edge weights is illustrated in Figure 1.1. We call

$$
\omega=\left\{R_{x}^{1}, R_{y}^{2},\left(Y_{z}^{1}, Y_{z}^{2}\right): x \in \mathbb{N} \times\{0\}, y \in\{0\} \times \mathbb{N}, z \in \mathbb{N}^{2}\right\}
$$

the polymer environment. We use $\mathbb{P}$ and $\mathbb{E}$ to denote the probability measure and corresponding expectation of the polymer environment.

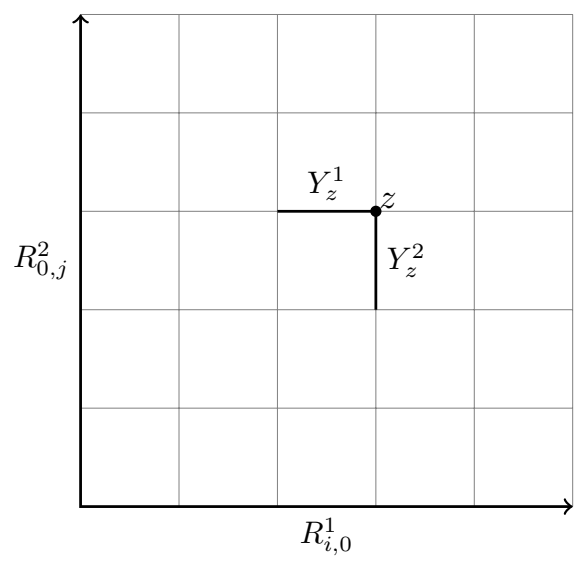

Figure 1.1. Assignment of edge weights. 
A path is weighted according to the product of the weights along its edges. For $(m, n) \in \mathbb{Z}_{+}^{2} \backslash\{(0,0)\}$ we define a probability measure on all up-right paths from $(0,0)$ to $(m, n)$. See Figure 1.2 for an example of an up-right path. Let $\Pi_{m, n}$ denote the collection of all such paths. We identify paths $x_{.}=\left(x_{0}, x_{1}, \ldots, x_{m+n}\right)$ by their sequence of vertices, but also associate to paths their sequence of edges $\left(e_{1}, \ldots, e_{m+n}\right)$, where $e_{i}=\left\{x_{i-1}, x_{i}\right\}$. Define the quenched polymer measure on $\Pi_{m, n}$,

$$
Q_{m, n}\left(x_{\bullet}\right):=\frac{1}{Z_{m, n}} \prod_{i=1}^{m+n} \omega_{e_{i}}
$$

where $\omega_{e}$ is the weight associated to the edge $e$ and

$$
Z_{m, n}:=\sum_{x, \in \Pi_{m, n}} \prod_{i=1}^{m+n} \omega_{e_{i}}
$$

is the associated partition function. At the origin, define $Z_{0,0}:=1$. Taking the expectation $\mathbb{E}$ of the quenched measure with respect to the edge weights gives the annealed measure on $\Pi_{m, n}$,

$$
P_{m, n}\left(x_{\bullet}\right):=\mathbb{E}\left[Q_{m, n}\left(x_{\bullet}\right)\right] .
$$

The annealed expectation will be denoted by $E_{m, n}$.

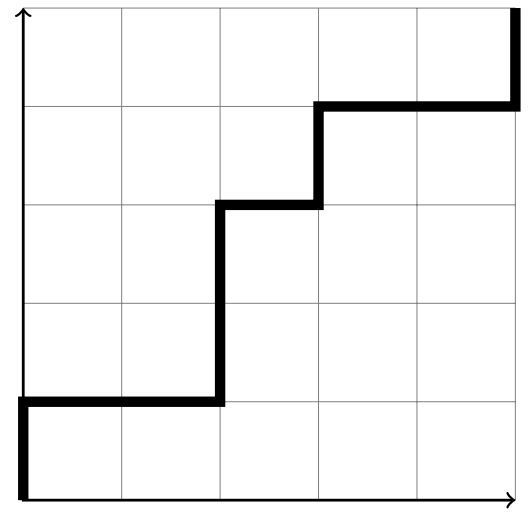

Figure 1.2. An up-right path from $(0,0)$ to $(5,5)$.

We specify the edge weight distributions for the four stationary polymer models. The notation $X \sim \mathrm{Ga}(\alpha, \beta)$ is used to denote that a random variable is gamma $(\alpha, \beta)$ distributed, i.e. has density $\Gamma(\alpha)^{-1} \beta^{\alpha} x^{\alpha-1} e^{-\beta x}$ supported on $(0, \infty)$, where $\Gamma(\alpha)=$ $\int_{0}^{\infty} x^{\alpha-1} e^{-x} d x$ is the gamma function. $X \sim \operatorname{Be}(\alpha, \beta)$ is used to say that $X$ is $\operatorname{beta}(\alpha, \beta)$ distributed, i.e. has density $\frac{\Gamma(\alpha+\beta)}{\Gamma(\alpha) \Gamma(\beta)} x^{\alpha-1}(1-x)^{\beta-1}$ supported on $(0,1)$. We then use $X \sim \mathrm{Ga}^{-1}(\alpha, \beta)$ and $X \sim \mathrm{Be}^{-1}(\alpha, \beta)$ to denote that $X^{-1} \sim \mathrm{Ga}(\alpha, \beta)$ and $X^{-1} \sim \operatorname{Be}(\alpha, \beta)$, respectively. We also use $X \sim\left(\operatorname{Be}^{-1}(\alpha, \beta)-1\right)$ to denote that $X+1 \sim \mathrm{Be}^{-1}(\alpha, \beta)$.

- Inverse-gamma (IG): This is also known as the log-gamma model. Assume $\mu>\theta>0, \beta>0$ and

$$
\begin{gathered}
R^{1} \sim \mathrm{Ga}^{-1}(\mu-\theta, \beta) \quad R^{2} \sim \mathrm{Ga}^{-1}(\theta, \beta) \\
\left(Y^{1}, Y^{2}\right)=(X, X) \quad \text { where } \quad X \sim \mathrm{Ga}^{-1}(\mu, \beta) .
\end{gathered}
$$


- Gamma (G): This is also known as the strict-weak model. Assume $\theta, \mu, \beta>0$ and

$$
\begin{gathered}
R^{1} \sim \mathrm{Ga}(\mu+\theta, \beta) \quad R^{2} \sim \mathrm{Be}^{-1}(\theta, \mu) \\
\left(Y^{1}, Y^{2}\right)=(X, 1) \quad \text { where } \quad X \sim \operatorname{Ga}(\mu, \beta) .
\end{gathered}
$$

- $\operatorname{Beta}(\mathbf{B})$ : Assume $\theta, \mu, \beta>0$ and

$$
\begin{gathered}
R^{1} \sim \operatorname{Be}(\mu+\theta, \beta) \quad R^{2} \sim \operatorname{Be}^{-1}(\theta, \mu) \\
\left(Y^{1}, Y^{2}\right)=(X, 1-X) \quad \text { where } \quad X \sim \operatorname{Be}(\mu, \beta) .
\end{gathered}
$$

- Inverse-beta (IB): Assume $\mu>\theta>0, \beta>0$ and

$$
\begin{array}{cr}
R^{1} \sim \mathrm{Be}^{-1}(\mu-\theta, \beta) & R^{2} \sim\left(\mathrm{Be}^{-1}(\theta, \beta+\mu-\theta)-1\right) \\
\left(Y^{1}, Y^{2}\right)=(X, X-1) & \text { where } \quad X \sim \operatorname{Be}^{-1}(\mu, \beta) .
\end{array}
$$

The name of each model refers to the distribution of the bulk weights. We call these models the four basic beta-gamma models.

1.2. Results. If $X$ is a positive random variable with density $\rho$, define

$$
L_{X}(x):=-\frac{1}{x \rho(x)} \operatorname{Cov}\left(\log X, \mathbb{1}_{\{X \leqslant x\}}\right)
$$

for all $x$ such that $\rho(x)>0$. Given a path $x . \in \Pi_{m, n}$, define the exit points of the path from the horizontal and vertical axes by

$$
t_{1}:=\max \left\{i:(i, 0) \in x_{\bullet}\right\} \quad \text { and } \quad t_{2}:=\max \left\{j:(0, j) \in x_{\bullet}\right\} .
$$

The following proposition gives exact formulas for the expectation and variance of the free energy, which is a starting point for analysis of these four models.

Proposition 1.1. Assume that the polymer environment has edge weight distributions $R^{1}, R^{2},\left(Y^{1}, Y^{2}\right)$ as in one of (1.2) through (1.5). Then for all $(m, n) \in \mathbb{Z}_{+}^{2}$,

$$
\mathbb{E}\left[\log Z_{m, n}\right]=m \mathbb{E}\left[\log R^{1}\right]+n \mathbb{E}\left[\log R^{2}\right],
$$

$$
\begin{aligned}
& \mathbb{V} a r\left[\log Z_{m, n}\right]=-m \mathbb{V} a r\left[\log R^{1}\right]+n \mathbb{V} \operatorname{ar}\left[\log R^{2}\right]+2 E_{m, n}\left[\sum_{i=1}^{t_{1}} L_{R^{1}}\left(R_{i, 0}^{1}\right)\right], \\
& \mathbb{V} \operatorname{ar}\left[\log Z_{m, n}\right]=m \mathbb{V} \operatorname{ar}\left[\log R^{1}\right]-n \mathbb{V} \operatorname{ar}\left[\log R^{2}\right]+2 E_{m, n}\left[\sum_{j=1}^{t_{2}} L_{R^{2}}\left(R_{0, j}^{2}\right)\right] .
\end{aligned}
$$

Using these exact formulas, we can obtain the following bounds on the variance of the free energy when $(m, n)$ grow in a characteristic direction.

Theorem 1.2. Assume that the polymer environment has edge weight distributions $R^{1}, R^{2},\left(Y^{1}, Y^{2}\right)$ as in one of $(1.2)$ through $(1.5)$, and let $(m, n)=\left(m_{N}, n_{N}\right)_{N=1}^{\infty}$ be a sequence such that

$$
\left|m_{N}-N \mathbb{V} a r\left[\log R^{2}\right]\right| \leqslant \gamma N^{2 / 3} \quad \text { and } \quad\left|n_{N}-N \mathbb{V} \operatorname{ar}\left[\log R^{1}\right]\right| \leqslant \gamma N^{2 / 3}
$$

for some fixed $\gamma>0$. Then there exist positive constants $c, C$, and $N_{0}$ depending only on $\mu, \theta, \beta, \gamma$ such that for all $N \geqslant N_{0}$,

$$
c N^{2 / 3} \leqslant \mathbb{V} a r\left[\log Z_{m, n}\right] \leqslant C N^{2 / 3} .
$$

The same constants $c, C, N_{0}$ can be taken for all $\mu, \theta, \beta, \gamma$ varying in a compact set. 
Theorem 1.2 and a Borel-Cantelli argument give the following law of large numbers.

Corollary 1.3. With assumptions as in Theorem 1.2 the following limit holds $\mathbb{P}$ almost surely

$$
\lim _{N \rightarrow \infty} \frac{\log Z_{m, n}}{N}=\mathbb{E}\left[\log R^{1}\right] \mathbb{V} \operatorname{ar}\left[\log R^{2}\right]+\mathbb{E}\left[\log R^{2}\right] \mathbb{V} \operatorname{ar}\left[\log R^{1}\right] .
$$

For the four basic beta-gamma models, the right-hand side of (1.11) is given by

$$
A(\mu, \theta, \beta)\left(\frac{\partial}{\partial \theta} B(\mu, \theta)\right)-\left(\frac{\partial}{\partial \theta} A(\mu, \theta, \beta)\right) B(\mu, \theta)+C(\mu, \theta),
$$

where the functions $A, B$, and $C$ are given in Table 1.3 and $\Psi_{n}(x):=\frac{\partial^{n+1}}{\partial x^{n+1}} \log \Gamma(x)$ denotes the polygamma function of order $n$.

\begin{tabular}{|l||c|c|c|}
\hline Model & $A(\mu, \theta, \beta)$ & $B(\mu, \theta)$ & $C(\mu, \theta)$ \\
\hline \hline IG & $\log \beta$ & $\Psi_{0}(\theta)-\Psi_{0}(\mu-\theta)$ & $-\Psi_{0}(\mu-\theta) \Psi_{1}(\theta)-\Psi_{0}(\theta) \Psi_{1}(\mu-\theta)$ \\
\hline G & $\log \beta$ & $\Psi_{0}(\mu+\theta)-\Psi_{0}(\theta)$ & $\Psi_{0}(\mu+\theta) \Psi_{1}(\theta)-\Psi_{0}(\theta) \Psi_{1}(\mu+\theta)$ \\
\hline B & $\Psi_{0}(\mu+\theta+\beta)$ & $\Psi_{0}(\mu+\theta)-\Psi_{0}(\theta)$ & $\Psi_{0}(\mu+\theta) \Psi_{1}(\theta)-\Psi_{0}(\theta) \Psi_{1}(\mu+\theta)$ \\
\hline IB & $-\Psi_{0}(\mu-\theta+\beta)$ & $\Psi_{0}(\theta)-\Psi_{0}(\mu-\theta)$ & $-\Psi_{0}(\mu-\theta) \Psi_{1}(\theta)-\Psi_{0}(\theta) \Psi_{1}(\mu-\theta)$ \\
\hline
\end{tabular}

Figure 1.3. Functions for the limiting rescaled free energy of the four basic beta-gamma models.

The following is a result for when the sequence $\left(m_{N}, n_{N}\right)$ does not satisfy condition (1.10). The statement is given for when the horizontal direction is too large, but an analogous result holds for the vertical direction.

Corollary 1.4. Assume that the polymer environment has edge weight distributions $R^{1}, R^{2},\left(Y^{1}, Y^{2}\right)$ as in one of (1.2) through (1.5) and that $m, n \rightarrow \infty$. Define $N$ by $n=N \mathbb{V} a r\left[\log R^{1}\right]$ and assume

$$
N^{-\alpha}\left(m-N \mathbb{V} a r\left[\log R^{2}\right]\right) \rightarrow c_{1}>0
$$

for some $\alpha>2 / 3$. Then as $N \rightarrow \infty$

$$
N^{-\alpha / 2}\left(\log Z_{m, n}-\mathbb{E}\left[\log Z_{m, n}\right]\right)
$$

converges in distribution to a centered normal with variance $c_{1} \mathbb{V}$ ar $\left[\log R^{1}\right]$.

The variance formulas in Proposition 1.1 connect the variance of the free energy to the exit points of the path from the boundaries (1.7). This allows us to obtain bounds on the polymer path fluctuations under the annealed measure.

Given a path $x_{\bullet} \in \Pi_{m, n}$, for $0 \leqslant k \leqslant m$ and $0 \leqslant l \leqslant n$ define

$$
\begin{aligned}
v_{0}(l) & :=\min \left\{i:(i, l) \in x_{.}\right\} & v_{1}(l) & :=\max \left\{i:(i, l) \in x_{.}\right\} \\
w_{0}(k) & :=\min \left\{j:(k, j) \in x_{.}\right\} & w_{1}(k) & :=\max \left\{j:(k, j) \in x_{.}\right\} .
\end{aligned}
$$

This is illustrated in Figure 1.4. 


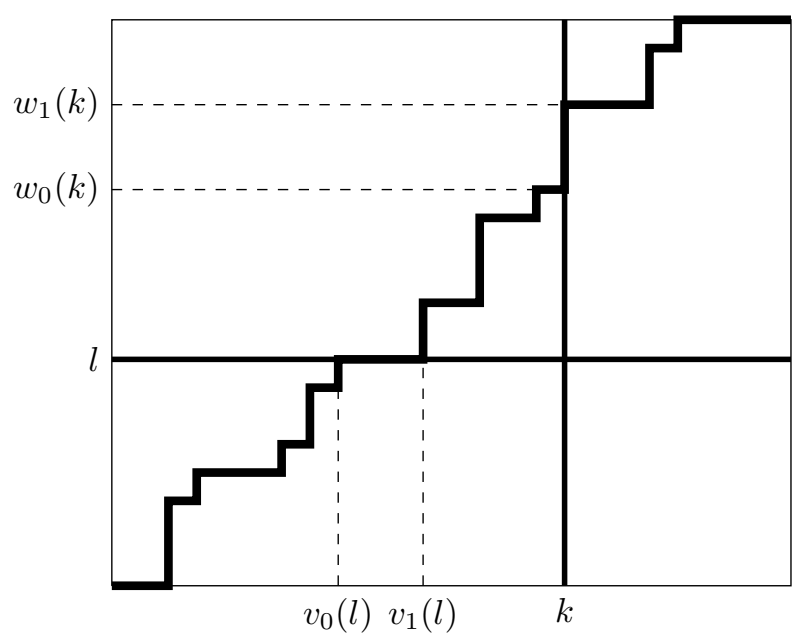

FiguRE 1.4. Example path with $v_{0}, v_{1}, w_{0}, w_{1}$ illustrated.

Theorem 1.5. Assume that the polymer environment has edge weight distributions $R^{1}, R^{2},\left(Y^{1}, Y^{2}\right)$ as in one of (1.2) through (1.5), and let $(m, n)=\left(m_{N}, n_{N}\right)_{N=1}^{\infty}$ be a sequence satisfying (1.10) for some fixed $\gamma>0$. Let $0 \leqslant \tau<1$. Then there exist positive constants $b_{0}, C, c_{0}, c_{1}, N_{0}$ depending only on $\mu, \theta, \beta, \gamma, \tau$ such that for $b \geqslant b_{0}$ and $N \in \mathbb{N}$,

$$
\begin{gathered}
P_{m, n}\left(v_{0}(\lfloor\tau n\rfloor) \leqslant \tau m-b N^{2 / 3} \text { or } v_{1}(\lfloor\tau n\rfloor) \geqslant \tau m+b N^{2 / 3}\right) \leqslant \frac{C}{b^{3}}, \\
P_{m, n}\left(w_{0}(\lfloor\tau m\rfloor) \leqslant \tau n-b N^{2 / 3} \text { or } w_{1}(\lfloor\tau m\rfloor) \geqslant \tau n+b N^{2 / 3}\right) \leqslant \frac{C}{b^{3}},
\end{gathered}
$$

and for all $N \geqslant N_{0}$,

$$
c_{0} \leqslant P_{m, n}\left(v_{1}(\lfloor\tau n\rfloor) \geqslant \tau m+c_{1} N^{2 / 3} \text { or } w_{1}(\lfloor\tau m\rfloor) \geqslant \tau n+c_{1} N^{2 / 3}\right) .
$$

The same constants can be taken for all $\mu, \theta, \beta, \gamma, \tau$ varying in a compact set.

Structure of the paper: In Section 2 we define the down-right property then state and prove consequences of this property. In Section 3 we introduce the Mellin transform framework, which allows us to treat the four basic beta-gamma models simultaneously, and prove Proposition 1.1. In Section 4 we prove the upper bound of Theorem 1.2. In Section 5 we prove bounds (1.13) and (1.14) of Theorem 1.5. In Section 6 we prove the lower bound of Theorem 1.2 and bound (1.15) of Theorem 1.5. In Appendix A we verify that each of the four basic beta-gamma models satisfies the conditions of Hypothesis 3.6. Appendix B collects technical lemmas used in Sections 3 and 4. Appendix C collects facts used in the proof of Proposition 6.1.

Acknowledgements: This work is part of our dissertation research at the University of Wisconsin-Madison. We thank our advisors Timo Seppäläinen and Benedek Valkó for their guidance and insights. 


\section{The down-right property}

Write $\alpha_{1}=(1,0), \alpha_{2}=(0,1)$. For $k=1,2$ define ratios of partition functions

$$
R_{x}^{k}:=\frac{Z_{x}}{Z_{x-\alpha_{k}}} \quad \text { for all } x \text { such that } x-\alpha_{k} \in \mathbb{Z}_{+}^{2} .
$$

Note that these extend the definitions of $R_{i, 0}^{1}$ and $R_{0, j}^{2}$, since for example $Z_{i, 0}=$ $\prod_{k=1}^{i} R_{k, 0}^{1}$. We say that $\pi=\left\{\pi_{k}\right\}_{k \in \mathbb{Z}}$ is a down-right path in $\mathbb{Z}_{+}^{2}$ if $\pi_{k} \in \mathbb{Z}_{+}^{2}$ and $\pi_{k+1}-\pi_{k} \in\left\{\alpha_{1},-\alpha_{2}\right\}$ for each $k \in \mathbb{Z}$. To each edge along a down-right path we associate the random variable

$$
\Lambda_{\left\{\pi_{k-1}, \pi_{k}\right\}}:= \begin{cases}R_{\pi_{k}}^{1} & \text { if }\left\{\pi_{k-1}, \pi_{k}\right\} \text { is horizontal, } \\ R_{\pi_{k-1}}^{2} & \text { if }\left\{\pi_{k-1}, \pi_{k}\right\} \text { is vertical. }\end{cases}
$$

The following definition is a weaker form of the Burke property (see Theorem 3.3 of Seppäläinen, 2012).

Definition 2.1. Say the polymer model has the down-right property if for all down-right paths $\pi=\left\{\pi_{k}\right\}_{k \in \mathbb{Z}}$, the random variables

$$
\Lambda(\pi):=\left\{\Lambda_{\left\{\pi_{k-1}, \pi_{k}\right\}}: k \in \mathbb{Z}\right\}
$$

are independent and each $R_{\pi_{k}}^{1}$ and $R_{\pi_{k}}^{2}$ appearing in the collection are respectively distributed as $R^{1}$ and $R^{2}$.

The partition functions satisfy the recurrence relation

$$
Z_{x}=Y_{x}^{1} Z_{x-\alpha_{1}}+Y_{x}^{2} Z_{x-\alpha_{2}} \quad \text { for } x \in \mathbb{N}^{2} .
$$

This recurrence relation then implies the recursions

$$
\begin{aligned}
& R_{x}^{1}=Y_{x}^{1}+Y_{x}^{2} \frac{R_{x-\alpha_{2}}^{1}}{R_{x-\alpha_{1}}^{2}} \quad \text { for } x \in \mathbb{N}^{2} . \\
& R_{x}^{2}=Y_{x}^{1} \frac{R_{x-\alpha_{1}}^{2}}{R_{x-\alpha_{2}}^{1}}+Y_{x}^{2}
\end{aligned}
$$

Using the recursions (2.2) we can reduce the down-right property to a simple preservation in distribution.

Lemma 2.2. Let $R^{1}, R^{2},\left(Y^{1}, Y^{2}\right)$ be positive random variables such that $R^{1}, R^{2}$ and the pair $\left(Y^{1}, Y^{2}\right)$ are independent. Put

$$
\left(\widetilde{R}^{1}, \widetilde{R}^{2}\right):=\left(Y^{1}+Y^{2} R^{1} / R^{2}, Y^{1} R^{2} / R^{1}+Y^{2}\right) .
$$

Then the polymer model with edge weights $R^{1}, R^{2},\left(Y^{1}, Y^{2}\right)$ has the down-right property if and only if $\left(\widetilde{R}^{1}, \widetilde{R}^{2}\right) \stackrel{d}{=}\left(R^{1}, R^{2}\right)$.

Proof of Lemma 2.2: Given a down-right path $\pi$, define its lower-left interior

$$
\operatorname{Int}(\pi):=\left\{x \in \mathbb{Z}_{+}^{2} \text { such that } x+(m, n) \in\{\pi\} \text { for some } m, n \in \mathbb{N}\right\} .
$$

If the polymer model with edge weights $R^{1}, R^{2},\left(Y^{1}, Y^{2}\right)$ has the down-right property, taking $\pi$ to be the unique down-right path with interior $\{(0,0)\}$ implies that $\left(R_{1,1}^{1}, R_{1,1}^{2}\right) \stackrel{d}{=}\left(R^{1}, R^{2}\right)$. Then $(2.2)$ and the fact that $\left(R_{1,0}^{1}, R_{0,1}^{2},\left(Y_{1,1}^{1}, Y_{1,1}^{2}\right)\right) \stackrel{d}{=}$ $\left(R^{1}, R^{2},\left(Y^{1}, Y^{2}\right)\right)$ imply that $\left(\widetilde{R}^{1}, \widetilde{R}^{2}\right) \stackrel{d}{=}\left(R^{1}, R^{2}\right)$. 
For the converse direction, we first prove the statement for $\pi$ with finite interior. The case when the interior is empty is true by assumption. Assume that the downright property holds for all paths $\pi$ with $|\operatorname{Int}(\pi)|=n$. Given a path $\pi$ with $|\operatorname{Int}(\pi)|=$ $n+1$ there exists $x$ such that $\pi$ traverses the right-down corner $\left\{x-\alpha_{1}, x, x-\alpha_{2}\right\}$. Let $\tilde{\pi}$ be the path which traverses the same points as $\pi$ with the exception of instead passing through the down-right corner $\left\{x-\alpha_{1}, x-\alpha_{1}-\alpha_{2}, x-\alpha_{2}\right\}$. Then $|\operatorname{Int}(\tilde{\pi})|=n$ and so $\left(R_{x-\alpha_{2}}^{1}, R_{x-\alpha_{1}}^{2}\right) \stackrel{d}{=}\left(R^{1}, R^{2}\right)$. Using (2.2), the assumption that $\left(\widetilde{R}^{1}, \widetilde{R}^{2}\right) \stackrel{d}{=}\left(R^{1}, R^{2}\right)$ and the independence of $\left(Y_{x}^{1}, Y_{x}^{2}\right)$ from the collection $\Lambda(\tilde{\pi})$ gives us that the collection $\Lambda(\pi)$ has the desired property.

To prove the statement for arbitrary $\pi$, pick a finite sub-collection $F$ of $\Lambda(\pi)$. Then there exists $\tilde{\pi}$ such that $\operatorname{Int}(\tilde{\pi})$ is finite and $F \subset \Lambda(\tilde{\pi})$. Since the statement holds for down-right paths with finite interior, we are done.

Proposition 2.3. Each of the four basic beta-gamma models, (1.2) through (1.5), possesses the down-right property.

Proof: The $\left(\widetilde{R}^{1}, \widetilde{R}^{2}\right) \stackrel{d}{=}\left(R^{1}, R^{2}\right)$ condition in Lemma 2.2 has been checked for the inverse-gamma, gamma, beta, and inverse-beta models by Lemma 3.2 of Seppäläinen (2012), Lemma 6.3 of Corwin et al. (2015), Lemma 3.1 of Balázs et al. (2018), and Proposition 3.1 of Thiery (2016) respectively.

The following lemma is an immediate consequence of the down-right property and the starting point for the proof of Proposition 1.1.

Lemma 2.4. If the polymer model with edge weights $R^{1}, R^{2},\left(Y^{1}, Y^{2}\right)$ possesses the down-right property and $\log R^{1}, \log R^{2}$ both have finite variance, then for all $(m, n) \in \mathbb{Z}_{+}^{2}$,

(a) $\mathbb{E}\left[\log Z_{m, n}\right]=m \mathbb{E}\left[\log R^{1}\right]+n \mathbb{E}\left[\log R^{2}\right]$,

(b) $\mathbb{V} a r\left[\log Z_{m, n}\right]=-m \mathbb{V} a r\left[\log R^{1}\right]+n \mathbb{V} \operatorname{ar}\left[\log R^{2}\right]+2 \mathbb{C o v}\left(S_{N}, S_{S}\right)$,

(c) $\mathbb{V} a r\left[\log Z_{m, n}\right]=m \mathbb{V} a r\left[\log R^{1}\right]-n \mathbb{V} a r\left[\log R^{2}\right]+2 \mathbb{C o v}\left(S_{E}, S_{W}\right)$,

$$
\begin{aligned}
& \text { where } \\
& \qquad \begin{aligned}
S_{N} & :=\log Z_{m, n}-\log Z_{0, n}=\sum_{i=1}^{m} \log R_{i, n}^{1}, \quad S_{S}
\end{aligned}:=\log Z_{m, 0}=\sum_{i=1}^{m} \log R_{i, 0}^{1}, \\
& S_{E}:=\log Z_{m, n}-\log Z_{m, 0}=\sum_{j=1}^{n} \log R_{m, j}^{2}, \quad S_{W}:=\log Z_{0, n}=\sum_{j=1}^{n} \log R_{0, j}^{2} .
\end{aligned}
$$

Proof: By the down-right property $S_{S}$ is independent of $S_{W}, S_{N}$ is independent of $S_{E}$, and

$$
\mathbb{V} \operatorname{ar}\left[S_{N}\right]=\operatorname{Var}\left[S_{S}\right]=m \mathbb{V} \operatorname{ar}\left[\log R^{1}\right], \quad \mathbb{V} \operatorname{ar}\left[S_{E}\right]=\mathbb{V a r}\left[S_{W}\right]=n \mathbb{V} \operatorname{ar}\left[\log R^{2}\right] .
$$

These facts along with the equalities $\log Z_{m, n}=S_{N}+S_{W}=S_{E}+S_{S}$ gives (a) and

$$
\begin{aligned}
\mathbb{V} \operatorname{ar}\left[\log Z_{m, n}\right] & =\mathbb{V} \operatorname{ar}\left[S_{N}\right]+\mathbb{V} \operatorname{ar}\left[S_{W}\right]+2 \mathbb{C o v}\left(S_{N}, S_{W}\right) \\
& =\mathbb{V} \operatorname{ar}\left[S_{N}\right]+\mathbb{V} \operatorname{ar}\left[S_{W}\right]+2 \mathbb{C o v}\left(S_{N}, S_{E}+S_{S}-S_{N}\right) \\
& =-\operatorname{Var}\left[S_{N}\right]+\mathbb{V} \operatorname{ar}\left[S_{W}\right]+2 \mathbb{C o v}\left(S_{N}, S_{S}\right) \\
& =-m \mathbb{V} \operatorname{ar}\left[\log R^{1}\right]+n \mathbb{V} \operatorname{ar}\left[\log R^{2}\right]+2 \mathbb{C o v}\left(S_{N}, S_{S}\right) .
\end{aligned}
$$

Similarly,

$$
\mathbb{V} \operatorname{ar}\left[\log Z_{m, n}\right]=-n \mathbb{V} \operatorname{ar}\left[\log R^{2}\right]+m \mathbb{V} \operatorname{ar}\left[\log R^{1}\right]+2 \mathbb{C o v}\left(S_{E}, S_{W}\right)
$$




\section{The Mellin transform framework}

In this section we develop a framework which allows us to treat the four basic beta-gamma models simultaneously.

Given a function $f:(0, \infty) \rightarrow[0, \infty)$, write $M_{f}$ for its Mellin transform

$$
M_{f}(a):=\int_{0}^{\infty} x^{a-1} f(x) d x
$$

for any $a \in \mathbb{R}$ such that the integral converges. Define

$$
D\left(M_{f}\right):=\operatorname{interior}\left(\left\{a \in \mathbb{R}: 0<M_{f}(a)<\infty\right\}\right) .
$$

Definition 3.1. Given a function $f:(0, \infty) \rightarrow[0, \infty)$ such that $D\left(M_{f}\right)$ is nonempty, we define a family of densities on $(0, \infty)$ parametrized by $a \in D\left(M_{f}\right)$ :

$$
\rho_{f, a}(x):=M_{f}(a)^{-1} x^{a-1} f(x) .
$$

We write $X \sim m_{f}(a)$ to denote that the random variable $X$ has density $\rho_{f, a}$.

Remark 3.2. If $f:(0, \infty) \rightarrow[0, \infty)$ is such that $D\left(M_{f}\right)$ is non-empty, then $M_{f}$ is $C^{\infty}$ throughout $D\left(M_{f}\right)$. Furthermore, if $X \sim m_{f}(a)$, then

(a) $\log X$ has finite exponential moments. That is, there exists some $\epsilon>0$ such that

$$
\mathbb{E}\left[e^{\epsilon|\log X|}\right] \leqslant \mathbb{E}\left[X^{\epsilon}\right]+\mathbb{E}\left[X^{-\epsilon}\right]=\frac{M_{f}(a+\epsilon)+M_{f}(a-\epsilon)}{M_{f}(a)}<\infty .
$$

(b) For all $k \in \mathbb{N}$,

$$
\frac{\partial^{k}}{\partial a^{k}} M_{f}(a)=M_{f}(a) \mathbb{E}\left[(\log X)^{k}\right] .
$$

(c) $\mathbb{E}[\log X]=\psi_{0}^{f}(a)$ and $\operatorname{Var}[\log X]=\psi_{1}^{f}(a)$, where

$$
\psi_{n}^{f}(a):=\frac{\partial^{n+1}}{\partial a^{n+1}} \log M_{f}(a) \text { for } n \in \mathbb{Z}_{+} .
$$

The following remark says that random variables with densities of the form (3.1) are closed under inversion.

Remark 3.3. If $f:(0, \infty) \rightarrow[0, \infty)$ is such that $D\left(M_{f}\right)$ is non-empty and $g(x):=$ $f\left(\frac{1}{x}\right)$ for $x \in(0, \infty)$, then for all $a \in D\left(M_{f}\right)$,

(a) $X \sim m_{f}(a) \Leftrightarrow X^{-1} \sim m_{g}(-a)$,

(b) $M_{f}(a)=M_{g}(-a)$ and therefore $D\left(M_{g}\right)=-D\left(M_{f}\right)$,

(c) $\psi_{n}^{f}(a)=(-1)^{n+1} \psi_{n}^{g}(-a)$ for all $n \in \mathbb{N}$.

Definition 3.4. Let $f^{j}:(0, \infty) \rightarrow[0, \infty)$ be such that $D\left(M_{f^{j}}\right)$ is non-empty for $j=1,2$. We say that the polymer environment is Mellin-type with respect to $\left(f^{1}, f^{2}\right)$ if $\left(R^{1}, R^{2}\right) \sim m_{f^{1}}\left(a_{1}\right) \otimes m_{f^{2}}\left(a_{2}\right)$ for some $a_{j} \in D\left(M_{f^{j}}\right)$.

When the polymer environment is Mellin-type with parameters $\left(a_{1}, a_{2}\right)$, we use $\mathbb{P}^{\left(a_{1}, a_{2}\right)}, \mathbb{E}^{\left(a_{1}, a_{2}\right)}, \operatorname{Var}^{\left(a_{1}, a_{2}\right)}, \operatorname{Cov}^{\left(a_{1}, a_{2}\right)}$ in place of $\mathbb{P}, \mathbb{E}, \mathbb{V a r}$, Cov respectively. 
3.1. The four basic beta-gamma models are Mellin-type. We first specify functions $f$ to obtain each of the random variables appearing in the four basic beta-gamma models. Note that the fourth column in Table 3.5 specifies the distribution of the random variable corresponding to $f$. We let $\mathrm{B}(a, b)=\frac{\Gamma(a) \Gamma(b)}{\Gamma(a+b)}$ denote the beta function and recall that $\Psi_{n}(x)=\frac{\partial^{n+1}}{\partial x^{n+1}} \log \Gamma(x)$. For the Table 3.5 we assume $b>0$ and $a \in D\left(M_{f}\right)$.

\begin{tabular}{|c|c|c|c|}
\hline$f(x)$ & $D\left(M_{f}\right)$ & $M_{f}(a)$ & $m_{f}(a)$ \\
\hline \hline$e^{-b x}$ & $(0, \infty)$ & $\Gamma(a) / b^{a}$ & $\mathrm{Ga}(a, b)$ \\
\hline$e^{-b / x}$ & $(-\infty, 0)$ & $\Gamma(-a) b^{a}$ & $\mathrm{Ga}^{-1}(-a, b)$ \\
\hline$(1-x)^{b-1} \mathbb{1}_{\{0<x<1\}}$ & $(0, \infty)$ & $\mathrm{B}(a, b)$ & $\mathrm{Be}(a, b)$ \\
\hline$\left(1-\frac{1}{x}\right)^{b-1} \mathbb{1}_{\{x>1\}}$ & $(-\infty, 0)$ & $\mathrm{B}(-a, b)$ & $\mathrm{Be}^{-1}(-a, b)$ \\
\hline$\left(\frac{x}{x+1}\right)^{b}$ & $(-b, 0)$ & $\mathrm{B}(-a, b+a)$ & $\mathrm{Be}^{-1}(-a, b+a)-1$ \\
\hline
\end{tabular}

\begin{tabular}{|c|c|}
\hline$f(x)$ & $\psi_{n}^{f}(a)$ \\
\hline \hline$e^{-b x}$ & $\Psi_{n}(a)-\delta_{n, 0} \log b$ \\
\hline$e^{-b / x}$ & $(-1)^{n+1}\left(\Psi_{n}(-a)-\delta_{n, 0} \log b\right)$ \\
\hline$(1-x)^{b-1} \mathbb{1}_{\{0<x<1\}}$ & $\Psi_{n}(a)-\Psi_{n}(a+b)$ \\
\hline$\left(1-\frac{1}{x}\right)^{b-1} \mathbb{1}_{\{x>1\}}$ & $(-1)^{n+1}\left(\Psi_{n}(-a)-\Psi_{n}(-a+b)\right)$ \\
\hline$\left(\frac{x}{x+1}\right)^{b}$ & $\Psi_{n}(a+b)+(-1)^{n+1} \Psi_{n}(-a)$ \\
\hline
\end{tabular}

FiguRE 3.5. Mellin framework data for the distributions appearing in the four basic beta-gamma models.

To express the distribution of the polymer environment in each of the four basic beta-gamma models given in (1.2) through (1.5) within this Mellin framework, we let

$$
\left(R^{1}, R^{2}, X\right) \sim m_{f^{1}}\left(a_{1}\right) \otimes m_{f^{2}}\left(a_{2}\right) \otimes m_{f^{1}}\left(a_{3}\right),
$$

where the functions $f^{1}, f^{2}$ and parameters $a_{j}, j=1,2,3$ are given in Table 3.6. Recall that in each of the models, $\left(Y^{1}, Y^{2}\right)$ are given in terms of $X$. For Table 3.6 we assume $\mu, \beta>0$.

\begin{tabular}{|l||c|c|c|c|}
\hline Model & $f^{1}(x)$ & $f^{2}(x)$ & $\left(a_{1}, a_{2}, a_{3}\right)$ & \\
\hline \hline IG & $e^{-\beta / x}$ & $e^{-\beta / x}$ & $(\theta-\mu,-\theta,-\mu)$ & $\theta \in(0, \mu)$ \\
\hline G & $e^{-\beta x}$ & $\left(1-\frac{1}{x}\right)^{\mu-1} \mathbb{1}_{\{x>1\}}$ & $(\mu+\theta,-\theta, \mu)$ & $\theta \in(0, \infty)$ \\
\hline B & $(1-x)^{\beta-1} \mathbb{1}_{\{0<x<1\}}$ & $\left(1-\frac{1}{x}\right)^{\mu-1} \mathbb{1}_{\{x>1\}}$ & $(\mu+\theta,-\theta, \mu)$ & $\theta \in(0, \infty)$ \\
\hline IB & $\left(1-\frac{1}{x}\right)^{\beta-1} \mathbb{1}_{\{x>1\}}$ & $\left(\frac{x}{x+1}\right)^{(\beta+\mu)}$ & $(\theta-\mu,-\theta,-\mu)$ & $\theta \in(0, \mu)$ \\
\hline
\end{tabular}

FIGURE 3.6. Functions and parameters to fit the four basic betagamma models into the Mellin framework.

Remark 3.5. For each fixed value of the bulk parameter $a_{3}$, we obtain a family of models with boundary parameters $a_{1}$ and $a_{2}$ satisfying $a_{1}+a_{2}=a_{3}$. For any such $a_{1}$ and $a_{2}$, by Proposition 2.3 these models will have the down-right property. 
3.2. Coupling of polymer environments. In order to compare polymer environments with different parameters, we use a coupling to express the boundary weights as functions of i.i.d. uniform $(0,1)$ random variables.

If $f:(0, \infty) \rightarrow[0, \infty)$ is such that $D\left(M_{f}\right)$ is non-empty, write $F^{f}$ for the CDF of the random variable $X \sim m_{f}(a)$. Specifically, $F^{f}: D\left(M_{f}\right) \times[0, \infty) \rightarrow[0,1]$ is given by

$$
F^{f}(a, x)=\frac{1}{M_{f}(a)} \int_{0}^{x} y^{a-1} f(y) d y .
$$

Define the quantile function

$$
H^{f}(a, p):=\inf \left\{x: p \leqslant F^{f}(a, x)\right\} .
$$

If the random variable $\eta$ is uniformly distributed on the interval $(0,1)$, then $H^{f}(a, \eta)$ $\sim m_{f}(a)$.

Suppose that a polymer environment $\omega$ is Mellin-type with respect to $\left(f^{1}, f^{2}\right)$ with parameters $\left(b_{1}, b_{2}\right)$. Let $\left\{\eta_{i}^{1}, \eta_{j}^{2}: i, j \in \mathbb{N}\right\}$ be i.i.d. uniform $(0,1)$ random variables that are independent of the bulk weights $\left\{\left(Y_{z}^{1}, Y_{z}^{2}\right): z \in \mathbb{N}^{2}\right\}$. Write $\widehat{\mathbb{P}}, \widehat{\mathbb{E}}$, and $\widehat{\mathbb{V a r}}$ for the probability measure and the corresponding expectation and variance of these uniform random variables and the bulk weights. Define the coupled environment

$$
\omega^{\left(b_{1}, b_{2}\right)}:=\left\{H^{f^{1}}\left(b_{1}, \eta_{i}^{1}\right), H^{f^{2}}\left(b_{2}, \eta_{j}^{2}\right),\left(Y_{z}^{1}, Y_{z}^{2}\right): i \in \mathbb{N}, j \in \mathbb{N}, z \in \mathbb{N}^{2}\right\} .
$$

Note that this environment is equal in distribution to the original environment $\omega$.

To specifically denote weights accumulated by a path, the partition function, the quenched measure, and the annealed expectation, associated to the coupled environment $\omega^{\left(b_{1}, b_{2}\right)}$, define

$$
\begin{aligned}
W\left(b_{1}, b_{2}\right)(x .) & :=\prod_{k=1}^{m+n} \omega_{\left(x_{k-1}, x_{k}\right)}^{\left(b_{1}, b_{2}\right)} \quad \text { for } x . \in \Pi_{m, n} \\
Z_{m, n}\left(b_{1}, b_{2}\right) & :=\sum_{x \cdot \in \Pi_{m, n}} W\left(b_{1}, b_{2}\right)\left(x_{\bullet}\right) \\
Q_{m, n}^{\left(b_{1}, b_{2}\right)}(A) & :=\frac{1}{Z_{m, n}\left(b_{1}, b_{2}\right)} \sum_{x, A} W\left(b_{1}, b_{2}\right)\left(x_{\bullet}\right) \quad \text { for } A \subset \Pi_{m, n} \\
E_{m, n}^{\left(b_{1}, b_{2}\right)}[\bullet] & :=\widehat{\mathbb{E}}\left[E^{\left.Q_{m, n}^{\left(b_{1}, b_{2}\right)}[\bullet]\right] .}\right.
\end{aligned}
$$

Recall the definition of the exit points $t_{j}(1.7)$. We can decompose the weight accumulated along a path to isolate the dependence on boundary weights

$$
W\left(b_{1}, b_{2}\right)\left(x_{\bullet}\right)=\prod_{i=1}^{t_{1}} H^{f^{1}}\left(b_{1}, \eta_{i}^{1}\right) \prod_{j=1}^{t_{2}} H^{f^{2}}\left(b_{2}, \eta_{j}^{2}\right) \prod_{k=\left(t_{1} \vee t_{2}\right)+1}^{m+n} \omega_{\left(x_{k}-1, x_{k}\right)}^{\left(b_{1}, b_{2}\right)} .
$$

Notice that one of the first two products will be empty and the third product involves only the bulk weights.

If we assume that $f:(0, \infty) \rightarrow[0, \infty)$ has open support, is continuous on its support, and $D\left(M_{f}\right)$ is non-empty, then $F^{f}$ is continuously differentiable on the set $D\left(M_{f}\right) \times \operatorname{supp}(f)$. By the implicit function theorem, $H^{f}$ is continuously differentiable and for all $(a, p) \in D\left(M_{f}\right) \times(0,1)$, we have

$$
\frac{\partial H^{f}}{\partial a}(a, p)=\frac{-\frac{\partial F^{f}}{\partial a}\left(a, H^{f}(a, p)\right)}{\frac{\partial F^{f}}{\partial x}\left(a, H^{f}(a, p)\right)}=H^{f}(a, p) L^{f}\left(a, H^{f}(a, p)\right)
$$


where $L^{f}$ is given by

$$
\begin{aligned}
L^{f}(a, x) & :=\frac{x^{-a}}{f(x)} \int_{0}^{x}\left(\psi_{0}^{f}(a)-\log y\right) y^{a-1} f(y) d y \\
& =-\frac{x^{-a}}{f(x)} \int_{x}^{\infty}\left(\psi_{0}^{f}(a)-\log y\right) y^{a-1} f(y) d y .
\end{aligned}
$$

The second equality follows from the definition of $\psi_{0}^{f}(a)$. Notice that

$$
L^{f}(a, x)=-\frac{1}{x \rho_{f, a}(x)} \operatorname{Cov}\left(\log X, \mathbb{1}_{\{X \leqslant x\}}\right)=L_{X}(x) \text { (as defined in (1.6)) }
$$

when $X \sim m_{f}(a)$, and therefore $L^{f}(a, x) \geqslant 0$.

The following hypothesis collects technical conditions for the function $f$ used in the sequel.

Hypothesis 3.6. Suppose that $f:(0, \infty) \rightarrow[0, \infty)$ is such that $D\left(M_{f}\right)$ is non-empty, $f$ has open support, is differentiable on its support, and for all compact $K \subset D\left(M_{f}\right)$ there exists a constant $C$ depending only on $K$ such that the following hold for all $a \in K$ :

$$
\begin{gathered}
L^{f}(a, x) \leqslant C(1+|\log x|) \quad \text { for all } x \in \operatorname{supp}(f), \\
\int_{0}^{1}\left|\frac{\partial}{\partial a} L^{f}\left(a, H^{f}(a, p)\right)\right| d p \leqslant C .
\end{gathered}
$$

Remark 3.7. If $X \sim m_{f}(a)$ where $f$ satisfies Hypothesis 3.6, then by (3.9) and Remark 3.2, $L_{X}(X)$ has finite exponential moments. By Lemma A.2 in the appendix, each of the functions $f$ corresponding to the random variables appearing in the four basic beta-gamma models (see Table 3.5) satisfies Hypothesis 3.6.

Lemma 3.8. Assume that the polymer environment is Mellin-type with respect to $\left(f^{1}, f^{2}\right)$, where $f^{1}$ and $f^{2}$ satisfy Hypothesis 3.6. Further assume that $\log Y^{1}$ and $\log Y^{2}$ have finite variance. Recall the notation (2.3). Then for all $(m, n) \in \mathbb{Z}_{+}^{2}$,

$$
\begin{aligned}
& \mathbb{C o v}\left(S_{N}, S_{S}\right)=E_{m, n}\left[\sum_{i=1}^{t_{1}} L_{R^{1}}\left(R_{i, 0}^{1}\right)\right], \\
& \mathbb{C o v}\left(S_{E}, S_{W}\right)=E_{m, n}\left[\sum_{j=1}^{t_{2}} L_{R^{2}}\left(R_{0, j}^{2}\right)\right] .
\end{aligned}
$$

Proof: By assumption, there exists $\left(a_{1}, a_{2}\right) \in D\left(M_{f^{1}}\right) \times D\left(M_{f^{2}}\right)$ such that $\left(R^{1}, R^{2}\right)$ $\sim m_{f^{1}}\left(a_{1}\right) \otimes m_{f^{2}}\left(a_{2}\right)$. There exist open neighborhoods $U_{j}$ about $a_{j}$ contained in $D\left(M_{f^{j}}\right)$ for $j=1,2$. We then show that

$$
\begin{aligned}
& \frac{\partial}{\partial b_{1}} \mathbb{E}^{\left(b_{1}, a_{2}\right)}\left[S_{N}\right]=\operatorname{Cov}^{\left(b_{1}, a_{2}\right)}\left(S_{N}, S_{S}\right) \text { for all } b_{1} \in U_{1}, \\
& \frac{\partial}{\partial b_{2}} \mathbb{E}^{\left(a_{1}, b_{2}\right)}\left[S_{E}\right]=\operatorname{Cov}^{\left(a_{1}, b_{2}\right)}\left(S_{E}, S_{W}\right) \text { for all } b_{2} \in U_{2},
\end{aligned}
$$

and that the mappings $b_{1} \mapsto \mathbb{C o v}^{\left(b_{1}, a_{2}\right)}\left(S_{N}, S_{S}\right)$ and $b_{2} \mapsto \mathbb{C o v}^{\left(a_{1}, b_{2}\right)}\left(S_{E}, S_{W}\right)$ are continuous. We begin with (3.13). We will vary the parameter $b_{1}$ of the weights $R_{i, 0}^{1}$ while keeping the parameter $a_{2}$ of the weights $R_{0, j}^{2}$ fixed. Let $\widetilde{\mathbb{E}}$ be the expectation

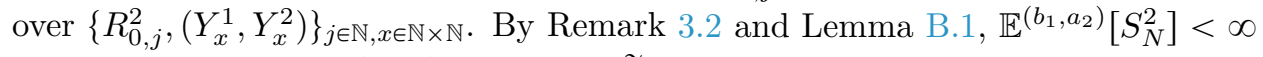
for all $b_{1} \in U_{1}$. Then $\mathbb{E}^{\left(b_{1}, a_{2}\right)}\left[S_{N}\right]=\mathbb{E}^{b_{1}}\left[\widetilde{\mathbb{E}}\left[S_{N}\right]\right]$ where $\mathbb{E}^{b_{1}}$ denotes the expectation 
over $\left\{R_{i, 0}^{1}\right\}_{i=1}^{m}$ when $R^{1} \sim m_{f^{1}}\left(b_{1}\right)$. We now invoke Lemma B.2. Specifically, we use $r=m, X_{k}=R_{k, 0}^{1}, f_{k}=f^{1}$ for all $k=1, \ldots, m$ and $A\left(R_{1,0}^{1}, \ldots, R_{m, 0}^{1}\right)=\widetilde{\mathbb{E}}\left[S_{N}\right]$ to get, for all $b_{1} \in U_{1}$,

$$
\begin{aligned}
\frac{\partial}{\partial b_{1}} \mathbb{E}^{\left(b_{1}, a_{2}\right)}\left[S_{N}\right]=\frac{\partial}{\partial b_{1}} \mathbb{E}^{b_{1}}\left[A\left(X_{1}, \ldots, X_{m}\right)\right] & =\operatorname{Cov}^{b_{1}}\left(A\left(X_{1}, \ldots, X_{m}\right), S_{S}\right) \\
& =\operatorname{Cov}^{\left(b_{1}, a_{2}\right)}\left(S_{N}, S_{S}\right)
\end{aligned}
$$

and $U_{1} \ni b_{1} \mapsto \mathbb{C o v}^{\left(b_{1}, a_{2}\right)}\left(S_{N}, S_{S}\right)$ is continuous. The third equality follows from the fact that the collection $\left\{R_{0, j}^{2},\left(Y_{x}^{1}, Y_{x}^{2}\right)\right\}_{j \in \mathbb{N}, x \in \mathbb{N} \times \mathbb{N}}$ is independent of $S_{S}$. The second moment condition of Lemma B.2 is satisfied since for all $b_{1} \in U_{1}$,

$$
\mathbb{E}^{b_{1}}\left[A\left(X_{1}, \ldots, X_{r}\right)^{2}\right]=\mathbb{E}^{b_{1}}\left[\left(\widetilde{\mathbb{E}}\left[S_{N}\right]\right)^{2}\right] \leqslant \mathbb{E}^{b_{1}}\left[\widetilde{\mathbb{E}}\left[S_{N}^{2}\right]\right]=\mathbb{E}^{\left(b_{1}, a_{2}\right)}\left[S_{N}^{2}\right]<\infty .
$$

A similar argument yields (3.14).

Using the coupling (3.4)

$$
E_{m, n}\left[\sum_{i=1}^{t_{1}} L_{R^{1}}\left(R_{i, 0}^{1}\right)\right]=E_{m, n}^{\left(a_{1}, a_{2}\right)}\left[\sum_{i=1}^{t_{1}} L^{f^{1}}\left(a_{1}, H^{f^{1}}\left(a_{1}, \eta_{i}^{1}\right)\right)\right] .
$$

Taking the derivative of (3.6) and using (3.7), for $j=1,2$

$$
\frac{\partial}{\partial b_{j}} \log \left(W\left(b_{1}, b_{2}\right)\left(x_{\bullet}\right)\right)=\sum_{k=1}^{t_{j}} \frac{\partial}{\partial b_{j}} \log H^{f^{j}}\left(b_{j}, \eta_{k}^{j}\right)=\sum_{k=1}^{t_{j}} L^{f^{j}}\left(b_{j}, H^{f^{j}}\left(b_{j} \eta_{k}^{j}\right)\right) .
$$

Therefore

$$
\frac{\partial}{\partial b_{j}} W\left(b_{1}, b_{2}\right)\left(x_{\bullet}\right)=W\left(b_{1}, b_{2}\right)\left(x_{\bullet}\right) \sum_{k=1}^{t_{j}} L^{f^{j}}\left(b_{j}, H^{f^{j}}\left(b_{j} \eta_{k}^{j}\right)\right)
$$

which implies that

$$
\frac{\partial}{\partial b_{j}} \log Z_{m, n}\left(b_{1}, b_{2}\right)=E^{Q_{m, n}^{\left(b_{1}, b_{2}\right)}}\left[\sum_{k=1}^{t_{j}} L^{f^{j}}\left(b_{j}, H^{f^{j}}\left(b_{j}, \eta_{k}^{j}\right)\right)\right] .
$$

We now prove (3.11). Similar to (3.5), in the coupled environment we use $S_{\bullet}\left(b_{1}, b_{2}\right)$ to make explicit the dependence of $S$. on the parameters $b_{1}$ and $b_{2}$. Recall that $\widehat{\mathbb{E}}$ is the expectation of the coupled environment. For $\epsilon>0$ small enough such that $\left[a_{1}-\epsilon, a_{1}+\epsilon\right] \subset U_{1}$,

$$
\begin{aligned}
\int_{a_{1}-\epsilon}^{a_{1}+\epsilon} \operatorname{Cov}^{\left(b_{1}, a_{2}\right)}\left(S_{N}, S_{S}\right) d b_{1} & =\mathbb{E}^{\left(a_{1}+\epsilon, a_{2}\right)}\left[S_{N}\right]-\mathbb{E}^{\left(a_{1}-\epsilon, a_{2}\right)}\left[S_{N}\right] \\
& =\widehat{\mathbb{E}}\left[S_{N}\left(a_{1}+\epsilon, a_{2}\right)-S_{N}\left(a_{1}-\epsilon, a_{2}\right)\right] \\
& =\widehat{\mathbb{E}}\left[\int_{a_{1}-\epsilon}^{a_{1}+\epsilon} \frac{\partial}{\partial b_{1}} \log Z_{m, n}\left(b_{1}, a_{2}\right) d b_{1}\right] \\
& =\int_{a_{1}-\epsilon}^{a_{1}+\epsilon} \widehat{\mathbb{E}}\left[\frac{\partial}{\partial b_{1}} \log Z_{m, n}\left(b_{1}, a_{2}\right)\right] d b_{1}
\end{aligned}
$$

where the first equality follows from (3.13), the third equality follows because $S_{W}$ does not depend on $b_{1}$ and $S_{N}\left(b_{1}, a_{2}\right)=\log Z_{m, n}\left(b_{1}, a_{2}\right)-S_{W}\left(a_{2}\right)$. The last equality follows from (3.18) and Tonelli's theorem (by the non-negativity of $L^{f^{1}}$ ). 
Recall that $b_{1} \mapsto \operatorname{Cov}^{\left(b_{1}, a_{2}\right)}\left(S_{N}, S_{S}\right)$ is continuous. Once we show that the mapping

$$
b_{1} \mapsto \widehat{\mathbb{E}}\left[\frac{\partial}{\partial b_{1}} \log Z_{m, n}\left(b_{1}, a_{2}\right)\right]=E_{m, n}^{\left(b_{1}, a_{2}\right)}\left[\sum_{i=1}^{t_{1}} L^{f^{1}}\left(b_{1}, H^{f^{1}}\left(b_{1}, \eta_{i}^{1}\right)\right)\right]
$$

is continuous, using (3.19) and (3.15) we will have (3.11). The continuity of (3.20) follows from the continuity of $b_{1} \mapsto E^{Q_{m, n}^{\left(b_{1}, a_{2}\right)}}\left[\sum_{k=1}^{t_{1}} L^{f^{1}}\left(b_{1}, H^{f^{1}}\left(b_{1}, \eta_{k}^{1}\right)\right)\right]$, the dominated convergence theorem, and the bound

$$
\begin{aligned}
& \widehat{\mathbb{E}}\left[\sup _{\left|b_{1}-a_{1}\right| \leqslant \epsilon} E^{Q_{m, n}^{\left(b_{1}, a_{2}\right)}}\left[\sum_{k=1}^{t_{1}} L^{f^{1}}\left(b_{1}, H^{f^{1}}\left(b_{1}, \eta_{k}^{1}\right)\right)\right]\right] \\
& \leqslant \widehat{\mathbb{E}}\left[\sup _{\left|b_{1}-a_{1}\right| \leqslant \epsilon} \sum_{k=1}^{m} L^{f^{1}}\left(b_{1}, H^{f^{1}}\left(b_{1}, \eta_{k}^{1}\right)\right)\right] \\
& \leqslant C \widehat{\mathbb{E}}\left[\sum_{k=1}^{m} 1+\left|\log H^{f^{1}}\left(a_{1}-\epsilon, \eta_{k}^{1}\right)\right|+\left|\log H^{f^{1}}\left(a_{1}+\epsilon, \eta_{k}^{1}\right)\right|\right]<\infty
\end{aligned}
$$

where we use the non-negativity of $L^{f^{1}}$ to replace $t_{1}$ by its upper bound $m$, then use assumption (3.9) of Hypothesis 3.6 (with the fact that $H^{f^{1}}(b, x)$ is non-decreasing in $b$ ) and part (a) of Remark 3.2.

A similar argument shows that

$$
\operatorname{Cov}^{\left(a_{1}, a_{2}\right)}\left(S_{E}, S_{W}\right)=E_{m, n}^{\left(a_{1}, a_{2}\right)}\left[\sum_{j=1}^{t_{2}} L^{f^{2}}\left(a_{2}, R_{0, j}^{2}\right)\right] .
$$

This completes the proof.

We can now give the proof of Proposition 1.1.

Proof of Proposition 1.1: By assumption, the polymer environment is distributed as in (3.2), where $f^{1}$ and $f^{2}$ satisfy Hypothesis 3.6 by Remark 3.7. By Remark 3.2, for each of the four models $\log u$ and $\log v$ have finite variance. Thus the conditions of Lemma 3.8 are satisfied. Combining Proposition 2.3 with Lemma 2.4, and Lemma 3.8 yields the result.

\section{Proof of the variance upper bound}

The first lemma of this section allows us to compare the variance of the free energy at different parameter values.

Lemma 4.1. Assume that the polymer environment is distributed as in (3.2). Let $\epsilon$ be small enough such that for all $|\lambda| \leqslant \epsilon, a_{1}+\lambda \in D\left(M_{f^{1}}\right)$ and $a_{2}-\lambda \in D\left(M_{f^{2}}\right)$. Then there exists a positive constant $C$ depending only on $\left(a_{1}, a_{2}\right), \beta$, and $\epsilon$ such that for all $(m, n) \in \mathbb{Z}_{+}^{2}$, the following holds for all $|\lambda| \leqslant \epsilon$,

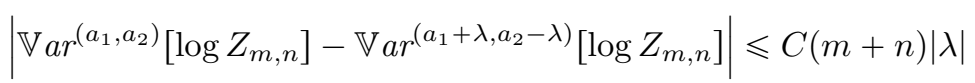

Proof: Let $\widetilde{a}_{1}=a_{1}+\lambda$ and $\widetilde{a}_{2}=a_{2}-\lambda$. Applying Proposition 1.1 (recalling that $\psi_{1}^{f}(a)=\operatorname{Var}[\log X]$ when $X \sim m_{f}(a)$ ) then using the coupling (3.5) yields, for $j=1,2$ : 


$$
\begin{aligned}
& \frac{1}{2}\left(\operatorname{Var}^{\left(\widetilde{a}_{1}, \widetilde{a}_{2}\right)}\left[\log Z_{m, n}\right]-\operatorname{Var}^{\left(a_{1}, a_{2}\right)}\left[\log Z_{m, n}\right]\right) \\
& =\frac{(-1)^{j}}{2}\left[m\left(\psi_{1}^{f^{1}}\left(\widetilde{a}_{1}\right)-\psi_{1}^{f^{1}}\left(a_{1}\right)\right)-n\left(\psi_{1}^{f^{2}}\left(\widetilde{a}_{2}\right)-\psi_{1}^{f^{2}}\left(a_{2}\right)\right)\right] \\
& \quad+E_{m, n}^{\left(\widetilde{a}_{1}, \widetilde{a}_{2}\right)}\left[\sum_{k=1}^{t_{j}} L^{f^{j}}\left(\widetilde{a}_{j}, H^{f^{j}}\left(\widetilde{a}_{j}, \eta_{k}^{j}\right)\right)\right]-E_{m, n}^{\left(a_{1}, a_{2}\right)}\left[\sum_{k=1}^{t_{j}} L^{f^{j}}\left(a_{j}, H^{f^{j}}\left(a_{j}, \eta_{k}^{j}\right)\right)\right] .
\end{aligned}
$$

Since $\psi_{1}^{f^{1}}$ and $\psi_{1}^{f^{2}}$ are continuously differentiable, there is a constant $C_{1}$ such that line (4.2) is bounded by $C_{1}(m+n)|\lambda|$. Suppressing the $m, n$ dependence, we then split line (4.3) as

$$
\begin{aligned}
= & \widehat{\mathbb{E}} E^{Q^{\left(\tilde{a}_{1}, \tilde{a}_{2}\right)}}\left[\sum_{k=1}^{t_{j}} L^{f^{j}}\left(\widetilde{a}_{j}, H^{f^{j}}\left(\widetilde{a}_{j}, \eta_{k}^{j}\right)\right)\right]-\widehat{\mathbb{E}} E^{Q^{\left(\tilde{a}_{1}, \tilde{a}_{2}\right)}}\left[\sum_{k=1}^{t_{j}} L^{f^{j}}\left(a_{j}, H^{f^{j}}\left(a_{j}, \eta_{k}^{j}\right)\right)\right] \\
& +\widehat{\mathbb{E}} E^{Q^{\left(\tilde{a}_{1}, \tilde{a}_{2}\right)}}\left[\sum_{k=1}^{t_{j}} L^{f^{j}}\left(a_{j}, H^{f^{j}}\left(a_{j}, \eta_{k}^{j}\right)\right)\right]-\widehat{\mathbb{E}} E^{Q^{\left(a_{1}, a_{2}\right)}}\left[\sum_{k=1}^{t_{j}} L^{f^{j}}\left(a_{j}, H^{f^{j}}\left(a_{j}, \eta_{k}^{j}\right)\right)\right]
\end{aligned}
$$

For line (4.4), since $t_{j}$ is all that is random under $E^{Q^{\left(\tilde{a}_{1}, \tilde{a}_{2}\right)}}$, we can replace $t_{j}$ by $m \vee n$. Thus

$$
\begin{aligned}
\mid \text { line (4.4)| } & \leqslant \widehat{\mathbb{E}} \sum_{k=1}^{m \vee n}\left|L^{f^{j}}\left(\widetilde{a}_{j}, H^{f^{j}}\left(\widetilde{a}_{j}, \eta_{k}^{j}\right)\right)-L^{f^{j}}\left(a_{j}, H^{f^{j}}\left(a_{j}, \eta_{k}^{j}\right)\right)\right| \\
& =(m \vee n) \int_{0}^{1}\left|L^{f^{j}}\left(\widetilde{a}_{j}, H^{f^{j}}\left(\widetilde{a}_{j}, \eta\right)\right)-L^{f^{j}}\left(a_{j}, H^{f^{j}}\left(a_{j}, \eta\right)\right)\right| d \eta \\
& =(m \vee n) \int_{0}^{1}\left|\int_{a_{j}}^{\widetilde{a}_{j}} \frac{\partial}{\partial a} L^{f^{j}}\left(a, H^{f^{j}}(a, \eta)\right) d a\right| d \eta \\
& \leqslant(m \vee n)\left|\int_{a_{j}}^{\tilde{a}_{j}} \int_{0}^{1}\right| \frac{\partial}{\partial a} L^{f^{j}}\left(a, H^{f^{j}}(a, \eta)\right)|d \eta d a| \\
& \leqslant(m \vee n) C_{2}|\lambda| .
\end{aligned}
$$

In the last step we used the fact that $f^{j}$ satisfy assumption (3.10) in Hypothesis 3.6 by Remark 3.7.

We can write line (4.5) as

$$
\widehat{\mathbb{E}}\left[\sum_{k=1}^{\ell_{j}} L^{f^{j}}\left(a_{j}, H^{f^{j}}\left(a_{j}, \eta_{k}^{j}\right)\right)\left(Q^{\left(\widetilde{a}_{1}, \tilde{a}_{2}\right)}\left(t_{j} \geqslant k\right)-Q^{\left(a_{1}, a_{2}\right)}\left(t_{j} \geqslant k\right)\right)\right],
$$

where $\ell_{1}=m$ and $\ell_{2}=n$. By Lemma B.3, $Q^{\left(a_{1}+\lambda, a_{2}-\lambda\right)}\left(t_{1} \geqslant k\right)$ is stochastically non-decreasing in $\lambda$, and $Q^{\left(a_{1}+\lambda, a_{2}-\lambda\right)}\left(t_{2} \geqslant k\right)$ is stochastically non-increasing in $\lambda$. Using the bound on (4.2), the bound (4.6), and the non-negativity of $L^{f^{j}}$, line (4.5) is non-negative if $j=1$ and $\lambda>0$ or $j=2$ and $\lambda<0$. This implies

$$
(4.1) \geqslant-C(m+n)|\lambda| \text {. }
$$


If $j=2$ and $\lambda>0$ or $j=1$ and $\lambda<0$, then (4.5) is non-positive, so

$$
(4.1) \leqslant C(m+n)|\lambda| \text {. }
$$

This completes the proof.

Lemma 4.2. Assume that the polymer environment is distributed as in (3.2). Then there exists a positive constant $C$ depending only on $\left(a_{1}, a_{2}\right)$ and $\beta$ such that for all $(m, n) \in \mathbb{Z}_{+}^{2}$ the following two inequalities hold:

$$
E_{m, n}\left[\sum_{i=1}^{t_{1}} L_{R^{1}}\left(R_{i, 0}^{1}\right)\right] \leqslant C\left(E_{m, n}\left[t_{1}\right]+1\right), \quad E_{m, n}\left[\sum_{j=1}^{t_{2}} L_{R^{2}}\left(R_{0, j}^{2}\right)\right] \leqslant C\left(E_{m, n}\left[t_{2}\right]+1\right) .
$$

Proof: Let $L_{i}=L_{R^{1}}\left(R_{i, 0}^{1}\right), \bar{L}_{i}=L_{i}-\mathbb{E}\left[L_{i}\right]$, and $S_{k}=\sum_{i=1}^{k} \bar{L}_{i}$. Note that $L_{i} \sim$ $L_{R^{1}}\left(R^{1}\right)$ has finite exponential moments by Remark 3.7. Using Cauchy-Schwarz, Markov's inequality, and the bound $\mathbb{E}\left[S_{k}^{8}\right] \leqslant C k^{4}$, we estimate

$$
\mathbb{E}\left[\mathbb{1}_{\left\{S_{k}>k\right\}} S_{k}\right] \leqslant\left(\mathbb{P}\left\{S_{k}>k\right\}\right)^{1 / 2}\left(k \mathbb{V a r} L_{1}\right)^{1 / 2} \leqslant\left(\frac{\mathbb{E}\left[S_{k}^{8}\right]}{k^{8}}\right)^{1 / 2}(k C)^{1 / 2} \leqslant C k^{-3 / 2} .
$$

Thus

Using this, we then get

$$
\sum_{k=1}^{\infty} \mathbb{E}\left[\mathbb{1}_{\left\{S_{k}>k\right\}} S_{k}\right] \leqslant C
$$

$$
\begin{aligned}
E_{m, n}\left[\sum_{i=1}^{t_{1}} L_{R^{1}}\left(R_{i, 0}^{1}\right)\right] & =E_{m, n}\left[\sum_{i=1}^{t_{1}} \bar{L}_{i}+\mathbb{E} L_{i}\right] \\
& =E_{m, n}\left[t_{1}\right] \mathbb{E}\left[L_{1}\right]+E_{m, n}\left[\sum_{i=1}^{t_{1}} \bar{L}_{i}\right] \\
& =E_{m, n}\left[t_{1}\right] \mathbb{E}\left[L_{1}\right]+\sum_{k=1}^{m} \mathbb{E}\left[Q_{m, n}\left(t_{1}=k\right) S_{k}\right] \\
& \leqslant E_{m, n}\left[t_{1}\right] \mathbb{E}\left[L_{1}\right]+\sum_{k=1}^{m}\left(k \mathbb{E}\left[Q_{m, n}\left(t_{1}=k\right)\right]+\mathbb{E}\left[\mathbb{1}_{\left\{S_{k}>k\right\}} S_{k}\right]\right) \\
& \leqslant E_{m, n}\left[t_{1}\right] \mathbb{E}\left[L_{1}\right]+E_{m, n}\left[t_{1}\right]+C \\
& \leqslant C\left(E_{m, n}\left[t_{1}\right]+1\right)
\end{aligned}
$$

The proof for $t_{2}$ is analogous.

Proposition 4.3. Assume that the polymer environment is distributed as in (3.2). Assume that the sequence $(m, n)=\left(m_{N}, n_{N}\right)_{N=1}^{\infty}$ satisfies

$$
\left|m-N \psi_{1}^{f^{2}}\left(a_{2}\right)\right| \vee\left|n-N \psi_{1}^{f^{1}}\left(a_{1}\right)\right| \leqslant \kappa_{N}
$$

where $\kappa_{N} \leqslant \gamma N^{2 / 3}$ and $\gamma$ is some positive constant.

Then there exist positive constants $C_{1}, C_{2}, C_{3}, \delta, \delta_{1}$ depending only on $\left(a_{1}, a_{2}\right), \beta$, and $\gamma$ such that for $N \in \mathbb{N}$ and $1 \vee C_{1} \kappa_{N} \leqslant u \leqslant \delta N$,

$$
\mathbb{P}\left\{Q_{m, n}\left(t_{j} \geqslant u\right) \geqslant e^{\frac{-\delta u^{2}}{N}}\right\} \leqslant C_{2}\left(\frac{N^{2}}{u^{4}} E_{m, n}\left[t_{j}\right]+\frac{N^{2}}{u^{3}}\right) \quad \text { for } j=1,2,
$$

while for $N \in \mathbb{N}$ and $u \geqslant 1 \vee C_{1} \kappa_{N} \vee \delta N$,

$$
\mathbb{P}\left\{Q_{m, n}\left(t_{j} \geqslant u\right) \geqslant e^{-\delta_{1} u}\right\} \leqslant 2 e^{-C_{3} u} \quad \text { for } j=1,2 .
$$


Proof: Let $\epsilon>0$ be small enough such that for all $|\lambda| \leqslant \epsilon, a_{1}(\lambda):=a_{1}+\lambda \in D\left(M_{f^{1}}\right)$ and $a_{2}(\lambda):=a_{2}-\lambda \in D\left(M_{f^{2}}\right)$. For the moment fix $\lambda_{1} \in[0, \epsilon], \lambda_{2} \in[-\epsilon, 0]$, and $u \geqslant 1$. The $\lambda_{j}$ will give the perturbation $\left(a_{1}\left(\lambda_{j}\right), a_{2}\left(\lambda_{j}\right)\right)$ of the parameters $\left(a_{1}, a_{2}\right)$ which will be used when dealing with the exit time $t_{j}$. Using the coupling in (3.5), (3.6) gives: for both $j=1,2$ and any path $x$. such that $t_{j}\left(x_{\bullet}\right) \geqslant u$,

$$
\frac{W\left(a_{1}, a_{2}\right)\left(x_{\bullet}\right)}{W\left(a_{1}\left(\lambda_{j}\right), a_{2}\left(\lambda_{j}\right)\right)\left(x_{\bullet}\right)}=\prod_{k=1}^{t_{j}} \frac{H^{f^{j}}\left(a_{j}, \eta_{k}^{j}\right)}{H^{f^{j}}\left(a_{j}\left(\lambda_{j}\right), \eta_{k}^{j}\right)} \leqslant \prod_{k=1}^{\lfloor u\rfloor} \frac{H^{f^{j}}\left(a_{j}, \eta_{k}^{j}\right)}{H^{f^{j}}\left(a_{j}\left(\lambda_{j}\right), \eta_{k}^{j}\right)},
$$

since $H^{f}(a, x)$ is non-decreasing in $a$. Therefore

$$
\begin{aligned}
Q_{m, n}^{\left(a_{1}, a_{2}\right)}\left(t_{j} \geqslant u\right) & =\frac{1}{Z_{m, n}\left(a_{1}, a_{2}\right)} \sum_{x \cdot \in \Pi_{m, n}} \mathbb{1}_{\{x . \geqslant u\}} W\left(a_{1}, a_{2}\right)\left(x_{\bullet}\right) \\
& \leqslant \frac{Z_{m, n}\left(a_{1}\left(\lambda_{j}\right), a_{2}\left(\lambda_{j}\right)\right)}{Z_{m, n}\left(a_{1}, a_{2}\right)} \prod_{k=1}^{\lfloor u\rfloor} \frac{H^{f^{j}}\left(a_{j}, \eta_{k}^{j}\right)}{H^{f^{j}}\left(a_{j}\left(\lambda_{j}\right), \eta_{k}^{j}\right)} .
\end{aligned}
$$

Then for all real numbers $z, r$

$$
\begin{aligned}
\mathbb{P}\left\{Q_{m, n}\left(t_{j} \geqslant u\right) \geqslant e^{-z}\right\} \leqslant & \widehat{\mathbb{P}}\left\{\prod_{k=1}^{\lfloor u\rfloor} \frac{H^{f^{j}}\left(a_{j}, \eta_{k}^{j}\right)}{H^{f^{j}}\left(a_{j}\left(\lambda_{j}\right), \eta_{k}^{j}\right)} \geqslant e^{-r}\right\} \\
& +\widehat{\mathbb{P}}\left\{\frac{Z_{m, n}\left(a_{1}\left(\lambda_{j}\right), a_{2}\left(\lambda_{j}\right)\right)}{Z_{m, n}\left(a_{1}, a_{2}\right)} \geqslant e^{r-z}\right\} .
\end{aligned}
$$

We now split the proof into two cases.

Case 1: $1 \vee C_{1} \kappa_{N} \leqslant u \leqslant \delta N$. Let $b, \delta>0$ be small enough such that $b \delta \leqslant \epsilon$. These constants will be determined through the course of the proof. Put $\lambda_{1}=\frac{b u}{N}$ and $\lambda_{2}=-\frac{b u}{N}$. The condition $u \leqslant \delta N$ guarantees that $-\epsilon \leqslant \lambda_{2}<0<\lambda_{1} \leqslant \epsilon$. Now plug in $r=\lfloor u\rfloor\left(\psi_{0}^{f^{j}}\left(a_{j}\left(\lambda_{j}\right)\right)-\psi_{0}^{f^{j}}\left(a_{j}\right)\right)-\frac{\delta u^{2}}{N}$ and $z=\frac{\delta u^{2}}{N}$ to obtain

$$
\text { RHS of }(4.7)=\widehat{\mathbb{P}}\left\{\sum_{k=1}^{\lfloor u\rfloor} \overline{\log H^{f^{j}}\left(a_{j}, \eta_{k}^{j}\right)-\log H^{f^{j}}\left(a_{j}\left(\lambda_{j}\right), \eta_{k}^{j}\right)} \geqslant \frac{\delta u^{2}}{N}\right\} \leqslant C \frac{N^{2}}{u^{3}}
$$

by Chebyshev's inequality and the fact that $H^{f}(a, \eta) \sim m_{f}(a)$. The constant $C$ here depends only on $\left(a_{1}, a_{2}\right), \epsilon$, and $\delta$. We will now show how to tune $b$ and $\delta$ as functions of $\left(a_{1}, a_{2}\right)$ and $\epsilon$ to get a meaningful bound on

$$
\begin{aligned}
(4.8)=\widehat{\mathbb{P}}\left\{\overline{\log Z_{m, n}\left(a_{1}\left(\lambda_{j}\right), a_{2}\left(\lambda_{j}\right)\right)}-\overline{\log Z_{m, n}\left(a_{1}, a_{2}\right)} \geqslant\right. \\
\left.\widehat{\mathbb{E}}\left[\log Z_{m, n}\left(a_{1}, a_{2}\right)-\log Z_{m, n}\left(a_{1}\left(\lambda_{j}\right), a_{2}\left(\lambda_{j}\right)\right)\right]+r-z\right\} .
\end{aligned}
$$

Since the parameters satisfy $a_{1}\left(\lambda_{j}\right)+a_{2}\left(\lambda_{j}\right)=a_{3}$, by Remark 3.5 , the down-right property is still satisfied for the perturbed model with parameters $\left(a_{1}\left(\lambda_{j}\right), a_{2}\left(\lambda_{j}\right)\right)$. 
Using Proposition 1.1 we can evaluate the right-hand side inside the above probability

$$
\begin{aligned}
= & m\left(\psi_{0}^{f^{1}}\left(a_{1}\right)-\psi_{0}^{f^{1}}\left(a_{1}\left(\lambda_{j}\right)\right)\right)+n\left(\psi_{0}^{f^{2}}\left(a_{2}\right)-\psi_{0}^{f^{2}}\left(a_{2}\left(\lambda_{j}\right)\right)\right) \\
& +\lfloor u\rfloor\left(\psi_{0}^{f^{j}}\left(a_{j}\left(\lambda_{j}\right)\right)-\psi_{0}^{f^{j}}\left(a_{j}\right)\right)-2 \delta \frac{u^{2}}{N} \\
= & \left(m-N \psi_{1}^{f^{2}}\left(a_{2}\right)\right)\left(\psi_{0}^{f^{1}}\left(a_{1}\right)-\psi_{0}^{f^{1}}\left(a_{1}\left(\lambda_{j}\right)\right)\right) \\
& +\left(n-N \psi_{1}^{f^{1}}\left(a_{1}\right)\right)\left(\psi_{0}^{f^{2}}\left(a_{2}\right)-\psi_{0}^{f^{2}}\left(a_{2}\left(\lambda_{j}\right)\right)\right) \\
& +N\left[\psi_{1}^{f^{2}}\left(a_{2}\right)\left(\psi_{0}^{f^{1}}\left(a_{1}\right)-\psi_{0}^{f^{1}}\left(a_{1}\left(\lambda_{j}\right)\right)\right)+\psi_{1}^{f^{1}}\left(a_{1}\right)\left(\psi_{0}^{f^{2}}\left(a_{2}\right)-\psi_{0}^{f^{2}}\left(a_{2}\left(\lambda_{j}\right)\right)\right)\right] \\
& +\lfloor u\rfloor\left(\psi_{0}^{f^{j}}\left(a_{j}\left(\lambda_{j}\right)\right)-\psi_{0}^{f^{j}}\left(a_{j}\right)\right)-2 \delta \frac{u^{2}}{N} \\
\geqslant & -\kappa_{N} \frac{b u}{N} C^{\prime}-N\left(\frac{b u}{N}\right)^{2} C^{\prime}+u\left(\frac{b u}{N}\right) C^{\prime \prime}-2 \delta \frac{u^{2}}{N} \\
= & \frac{u}{N}\left[C^{\prime \prime} b u-C^{\prime} b^{2} u-2 \delta u-C^{\prime} b \kappa_{N}\right]
\end{aligned}
$$

for some positive constants $C^{\prime}$ and $C^{\prime \prime}$. This can be obtained by taking a 2nd-order Taylor expansion of the functions $\psi_{0}^{f^{j}}$, keeping in mind that $\psi_{1}^{f^{j}}>0$. In the last inequality we also used $u \geqslant 1$.

Now fixing $b$ small enough followed by then fixing $\delta$ small enough we can ensure that the entire quantity (4.11) is $\geqslant C^{\prime \prime \prime} \frac{u^{2}}{N}$ for some positive constant $C^{\prime \prime \prime}$ as long as $u \geqslant C_{1} \kappa_{N}$ for some positive $C_{1}$. With these restrictions,

$$
\begin{aligned}
(4.8) & \leqslant \widehat{\mathbb{P}}\left\{\widehat{\log Z_{m, n}\left(a_{1}\left(\lambda_{j}\right), a_{2}\left(\lambda_{j}\right)\right)-\log Z_{m, n}\left(a_{1}, a_{2}\right)} \geqslant C^{\prime \prime \prime} \frac{u^{2}}{N}\right\} \\
& \leqslant \frac{N^{2}}{\left(C^{\prime \prime \prime}\right)^{2} u^{4}} \widehat{\operatorname{Var}}\left[\log Z_{m, n}\left(a_{1}\left(\lambda_{j}\right), a_{2}\left(\lambda_{j}\right)\right)-\log Z_{m, n}\left(a_{1}, a_{2}\right)\right] \\
& \leqslant C \frac{N^{2}}{u^{4}}\left(\widehat{\operatorname{Var}}\left[\log Z_{m, n}\left(a_{1}, a_{2}\right)\right]+(m+n) \frac{b u}{N}\right) \\
& \leqslant C\left(\frac{N^{2}}{u^{4}} E_{m, n}\left[t_{j}\right]+\frac{N^{2}}{u^{3}}\right) .
\end{aligned}
$$

The second to last and last inequalities are applications of Lemma 4.1, Proposition 1.1, and Lemma 4.2. Combining this result with (4.9) finishes the first case.

Case 2: $1 \vee C_{1} \kappa_{N} \vee \delta N \leqslant u$. Take $\delta, \epsilon$ fixed from the first case, let $\delta_{1} \in(0, \delta]$, and $\epsilon_{1} \in(0, \epsilon]$. The constants $\delta_{1}$ and $\epsilon_{1}$ will be determined throughout the course of the proof. This time, put $\lambda_{1}=\epsilon_{1}, \lambda_{2}=-\epsilon_{1}, r=\lfloor u\rfloor\left(\psi_{0}^{f^{j}}\left(a_{j}\left(\lambda_{j}\right)\right)-\psi_{0}^{f^{j}}\left(a_{j}\right)\right)-\delta_{1} u$, and $z=\delta_{1} u$. Then

$$
(4.7)=\widehat{\mathbb{P}}\left\{\sum_{k=1}^{\lfloor u\rfloor} \overline{\left.\log H^{f^{j}}\left(a_{j}, \eta_{k}^{j}\right)-\log H^{f^{j}}\left(a_{j}\left(\lambda_{j}\right), \eta_{k}^{j}\right)\right)} \geqslant \delta_{1} u\right\} .
$$

By Remark 3.2 the random variables in the summation have finite exponential moments. A large deviation estimate gives us the existence of a positive constant $C_{3}$ such that $(4.12) \leqslant e^{-C_{3} u}$. 
We now consider (4.10). A similar analysis to that in Case 1 tells us that the right-hand side inside of the above probability

$$
\begin{aligned}
& \geqslant-C^{\prime} \epsilon_{0} \kappa_{N}-C^{\prime} \epsilon_{0}^{2} N+C^{\prime \prime} \epsilon_{0} u-2 \delta_{1} u \\
& \geqslant u\left(C^{\prime \prime} \epsilon_{0}-\frac{C^{\prime} \epsilon_{0}^{2}}{\delta}-2 \delta_{1}\right)-C^{\prime} \epsilon_{0} \kappa_{N}
\end{aligned}
$$

for some positive constants $C^{\prime}$ and $C^{\prime \prime}$ (the second inequality follows from $u \geqslant \delta N$ ). Now fixing $\epsilon_{0}$ small enough followed by then fixing $\delta_{1}$ small enough we can ensure that (4.13) $\geqslant C u$ for some positive constant $C$ as long as $u \geqslant C_{1} \kappa_{N}$ for some positive $C_{1}$ (here we increase the previous constant $C_{1}$ found in Case 1 if necessary). With these constraints,

$$
(4.8) \leqslant \widehat{\mathbb{P}}\left\{\overline{\log Z_{m, n}\left(a_{1}\left(\lambda_{j}\right), a_{2}\left(\lambda_{j}\right)\right)-\log Z_{m, n}\left(a_{1}, a_{2}\right)} \geqslant C u\right\} .
$$

Since the perturbed parameters are such that the polymer environment still has the down-right property, the random variable inside the above probability can be expressed as two sums of i.i.d. random variables, each of which has entries with finite exponential moments. Therefore a large deviation estimate gives the existence of a positive constant $C_{3}$ such that $(4.8) \leqslant e^{-u C_{3}}$. Combining this with (4.12) completes the proof.

Remark 4.4. If $\epsilon>0$ is small enough such that for all $|\lambda| \leqslant \epsilon, a_{1}+\lambda \in D\left(M_{f^{1}}\right)$ and $a_{2}-\lambda \in D\left(M_{f^{2}}\right)$, then the constants in Proposition 4.3 can be chosen such that the conclusion also holds for the polymer environment with parameters $\left(a_{1}+\right.$ $\left.\lambda, a_{2}-\lambda, a_{3}\right)$ for any $|\lambda| \leqslant \epsilon$.

Using the previous proposition, we can now bound the annealed expectation of the exit points of the polymer path from the axes.

Corollary 4.5. Suppose all of the assumptions of Proposition 4.3 hold. Then there exists a positive constant $C$ depending only on $\left(a_{1}, a_{2}\right), \beta$, and $\gamma$ such that for both $j=1,2$,

$$
E_{m, n}\left[t_{j}\right] \leqslant C N^{2 / 3} \quad \text { for all } N \in \mathbb{N} \text {. }
$$

Proof of Corollary 4.5: Since all of the constants $C_{1}, C_{2}, C_{3}, \delta, \delta_{1}$ determined by Proposition 4.3 depend only on $\left(a_{1}, a_{2}\right), \beta$, and $\gamma$, it is sufficient to show that the constant $C$ to be determined in this proof depends only on these five constants and $\gamma$. Let $r \geqslant 1 \vee C_{1} \gamma$. Then $r N^{2 / 3} \geqslant 1 \vee C_{1} \kappa_{N}$. Suppressing the $m, n$ dependence,

$$
\begin{aligned}
E\left[t_{j}\right] & =\int_{0}^{\infty} P\left(t_{j} \geqslant u\right) d u \\
& \leqslant r N^{2 / 3}+\int_{r N^{2 / 3}}^{r N^{2 / 3} \vee \delta N} P\left(t_{j} \geqslant u\right) d u+\int_{r N^{2 / 3} \vee \delta N}^{\infty} P\left(t_{j} \geqslant u\right) d u .
\end{aligned}
$$


We now bound the integrals in line (4.14) individually.

$$
\begin{aligned}
\int_{r N^{2 / 3} \vee \delta N}^{\infty} P\left(t_{j} \geqslant u\right) d u= & \int_{r N^{2 / 3} \vee \delta N}^{\infty} \int_{0}^{e^{-\delta_{1} u}} \mathbb{P}\left\{Q\left(t_{j} \geqslant u\right) \geqslant x\right\} d x d u \\
& +\int_{r N^{2 / 3} \vee \delta N}^{\infty} \int_{e^{-\delta_{1} u}}^{1} \mathbb{P}\left\{Q\left(t_{j} \geqslant u\right) \geqslant x\right\} d x d u . \\
\leqslant & \int_{\delta N}^{\infty} e^{-\delta_{1} u} d u \\
& +\int_{r N^{2 / 3} \vee \delta N}^{\infty} \int_{0}^{\delta_{1}} \mathbb{P}\left\{Q\left(t_{j} \geqslant u\right) \geqslant e^{-s u}\right\} e^{-s u} u d s d u \\
\leqslant & \frac{1}{\delta_{1}} e^{-\delta_{1} \delta N}+\int_{r N^{2 / 3} \vee \delta N}^{\infty} \int_{0}^{\delta_{1}} 2 e^{-\left(C_{3}+s\right) u} u d s d u \leqslant C,
\end{aligned}
$$

where in the first inequality we bounded the first integrand by one and made the substitution $x=e^{-s u}$ for the second. For the second inequality, we apply Proposition 4.3 to get that $\mathbb{P}\left\{Q\left(t_{j} \geqslant u\right) \geqslant e^{-s u}\right\} \leqslant \mathbb{P}\left\{Q\left(t_{j} \geqslant u\right) \geqslant e^{-\delta_{1} u}\right\} \leqslant 2 e^{-C_{3} u}$ for all $u \geqslant r N^{2 / 3} \vee \delta N$ and all $0<s \leqslant \delta_{1}$.

We now bound the first integral of (4.14). Without loss of generality, assume that $r N^{2 / 3}<\delta N$. Then

$$
\begin{aligned}
\int_{r N^{2 / 3}}^{r N^{2 / 3} \vee \delta N} P\left(t_{j} \geqslant u\right) d u= & \int_{r N^{2 / 3}}^{\delta N} \int_{0}^{e^{-\delta \frac{u^{2}}{N}}} \mathbb{P}\left\{Q\left(t_{j} \geqslant u\right) \geqslant x\right\} d x d u \\
& +\int_{r N^{2 / 3}}^{\delta N} \int_{e^{-\delta \frac{u^{2}}{N}}}^{1} \mathbb{P}\left\{Q\left(t_{j} \geqslant u\right) \geqslant x\right\} d x d u \\
\leqslant & \int_{r N^{2 / 3}}^{\delta N} e^{-\delta \frac{u^{2}}{N}} d u \\
& +\int_{r N^{2 / 3}}^{\delta N} \int_{0}^{\delta} \mathbb{P}\left\{Q\left(t_{j} \geqslant u\right) \geqslant e^{-s \frac{u^{2}}{N}}\right\} e^{-s \frac{u^{2}}{N}} \frac{u^{2}}{N} d s d u \\
\leqslant & \delta N e^{-\delta r^{2} N^{1 / 3}}+\int_{r N^{2 / 3}}^{\infty} \int_{e^{-s \frac{u^{2}}{N}}}^{1} C_{2}\left(\frac{N^{2}}{u^{4}} E\left[t_{j}\right]+\frac{N^{2}}{u^{3}}\right) d s d u \\
\leqslant & C+C_{2}\left(\frac{E\left[t_{j}\right]}{3 r^{3}}+\frac{N^{2 / 3}}{2 r^{2}}\right)
\end{aligned}
$$

where for the first inequality we bound the first integrand by one and make the substitution $x=e^{-s \frac{u^{2}}{N}}$ for the second. For the second inequality we apply Proposition 4.3 to get that $\mathbb{P}\left\{Q\left(t_{j} \geqslant u\right) \geqslant e^{-s \frac{u^{2}}{N}}\right\} \leqslant \mathbb{P}\left\{Q\left(t_{j} \geqslant u\right) \geqslant e^{-\delta \frac{u^{2}}{N}}\right\} \leqslant$ $C_{2}\left(\frac{N^{2}}{u^{4}} E\left[t_{j}\right]+\frac{N^{2}}{u^{3}}\right)$ for all $r N^{2 / 3} \leqslant u \leqslant \delta N$ and all $0<s \leqslant \delta$.

Combining the bounds on (4.15), (4.16) and (4.14), we have: for all $r \geqslant 1 \vee C_{1} \gamma$,

$$
E\left[t_{j}\right] \leqslant r N^{2 / 3}+C+C_{2}\left(\frac{E\left[t_{j}\right]}{3 r^{3}}+\frac{N^{2 / 3}}{2 r^{2}}\right) .
$$

We can now fix $r$ large enough with respect to $C$ and $C_{2}$ then rearrange to get the desired result.

We can now give the proof of the upper bound of the variance of the free energy. 
Proof of upper bound of Theorem 1.2: Averaging (1.8) and (1.9) of Proposition 1.1 then applying Lemma 4.2 followed by Corollary 4.5 (recalling that $\psi_{1}^{f^{j}}\left(a_{j}\right)=$ $\left.\operatorname{Var}\left[\log R^{j}\right]\right)$ gives

$$
\begin{aligned}
\operatorname{Var}\left[\log Z_{m, n}\right] & =E_{m, n}\left[\sum_{i=1}^{t_{1}} L_{R^{1}}\left(R_{i, 0}^{1}\right)\right]+E_{m, n}\left[\sum_{j=1}^{t_{2}} L_{R^{2}}\left(R_{0, j}^{2}\right)\right] \\
& \leqslant C\left(E_{m, n}\left[t_{1}\right]+E_{m, n}\left[t_{2}\right]+2\right) \\
& \leqslant C N^{2 / 3}
\end{aligned}
$$

which concludes the proof.

The following corollary is obtained by combining Proposition 4.3 and Corollary 4.5 .

Corollary 4.6. Assume that the polymer environment is distributed as in (3.2) and the sequence $(m, n)=\left(m_{N}, n_{N}\right)_{N=1}^{\infty}$ satisfies (1.10) for some positive constant $\gamma$. Then there exists positive constants $b_{0}, C_{2}, C_{3}, \delta$, and $\delta_{1}$ depending only on $\left(a_{1}, a_{2}\right), \beta$, and $\gamma$ such that for all $N \in \mathbb{N}$ and $b_{0} \leqslant b \leqslant \delta N^{1 / 3}$,

$$
\mathbb{P}\left\{Q_{m, n}\left(t_{j} \geqslant b N^{2 / 3}\right) \geqslant e^{-\delta b^{2} N^{1 / 3}}\right\} \leqslant \frac{2 C_{2}}{b^{3}} \quad \text { for } j=1,2,
$$

while for all $N \in \mathbb{N}$ and $b \geqslant b_{0} \vee \delta N^{1 / 3}$,

$$
\mathbb{P}\left\{Q_{m, n}\left(t_{j} \geqslant b N^{2 / 3}\right) \geqslant e^{-\delta_{1} b N^{2 / 3}}\right\} \leqslant 2 e^{-C_{3} b N^{2 / 3}} \quad \text { for } j=1,2 .
$$

Lemma 4.7. Assume that the polymer environment is distributed as in (3.2) and the sequence $(m, n)=\left(m_{N}, n_{N}\right)_{N=1}^{\infty}$ satisfies (1.10) for some positive constant $\gamma$. Then there exist constants $b_{0} \geqslant 1$ and $C>0$ depending only on $\left(a_{1}, a_{2}\right), \beta$, and $\gamma$ such that for all $b \geqslant b_{0}$ and $N \in \mathbb{N}$,

$$
P_{m, n}\left(t_{j} \geqslant b N^{2 / 3}\right) \leqslant \frac{C}{b^{3}} \quad \text { for } j=1,2 .
$$

Therefore, for all $0<p<3$ there exists a positive constant $C^{\prime}$ depending on $\left(a_{1}, a_{2}\right), \beta, \gamma$, and $p$ such that for all $N \in \mathbb{N}$,

$$
E_{m, n}\left[\left(\frac{t_{j}}{N^{2 / 3}}\right)^{p}\right] \leqslant C^{\prime} \quad \text { for } j=1,2 .
$$

Proof of Lemma 4.7: By Corollary (4.6) there exist positive constants $b_{0}, C_{2}, C_{3}, \delta$, $\delta_{1}$ with $b_{0} \geqslant 1$ such that (4.17) holds for $b_{0} \leqslant b \leqslant \delta N^{1 / 3}$ while (4.18) holds for $b \geqslant \delta N^{1 / 3} \vee b_{0}$.

We first estimate for $b \leqslant \delta N^{1 / 3}$,

$$
\begin{aligned}
P_{m, n}\left(t_{j} \geqslant b N^{2 / 3}\right)= & \int_{0}^{1} \mathbb{P}\left\{Q_{m, n}\left(t_{j} \geqslant b N^{2 / 3}\right) \geqslant x\right\} d x \\
= & \int_{0}^{\delta} \mathbb{P}\left\{Q_{m, n}\left(t_{j} \geqslant b N^{2 / 3}\right) \geqslant e^{-s b^{2} N^{1 / 3}}\right\} b^{2} N^{1 / 3} e^{-s b^{2} N^{1 / 3}} d s \\
& \quad+\int_{\delta}^{\infty} \mathbb{P}\left\{Q_{m, n}\left(t_{j} \geqslant b N^{2 / 3}\right) \geqslant e^{-s b^{2} N^{1 / 3}}\right\} b^{2} N^{1 / 3} e^{-s b^{2} N^{1 / 3}} d s \\
\leqslant & \frac{2 C_{2}}{b^{3}}+e^{-\delta b^{2} N^{1 / 3}} \leqslant \frac{C}{b^{3}}
\end{aligned}
$$


for some positive constant $C$, where we made the substitution $x=e^{-s b^{2} N^{1 / 3}}$, used (4.17) to bound the probability inside the integral of (4.19), and bounded the probability inside the integral of (4.20) by 1 . For $b \geqslant \delta N^{1 / 3}$, we make the substitution $x=e^{-s b N^{2 / 3}}$ to get

$$
\begin{aligned}
P_{m, n}\left(t_{j} \geqslant b N^{2 / 3}\right)= & \int_{0}^{1} \mathbb{P}\left\{Q_{m, n}\left(t_{j} \geqslant b N^{2 / 3}\right) \geqslant x\right\} d x \\
= & \int_{0}^{\delta_{1}} \mathbb{P}\left\{Q_{m, n}\left(t_{j} \geqslant b N^{2 / 3}\right) \geqslant e^{-s b N^{2 / 3}}\right\} b N^{2 / 3} e^{-s b N^{2 / 3}} d s \\
& \quad+\int_{\delta_{1}}^{\infty} \mathbb{P}\left\{Q_{m, n}\left(t_{j} \geqslant b N^{2 / 3}\right) \geqslant e^{-s b N^{2 / 3}}\right\} b N^{2 / 3} e^{-s b N^{2 / 3}} d s \\
\leqslant & 2 e^{-C_{3} b N^{2 / 3}}+e^{-\delta_{1} b N^{2 / 3}} \leqslant \frac{C}{b^{3}}
\end{aligned}
$$

increasing the constant $C$ if necessary, where we used (4.18) to bound the probability inside the integral of (4.21) and bounded the probability inside the integral of (4.22) by 1 .

Proof of Corollary 1.4: Let $m_{1}=\left\lfloor N \mathbb{V a r}\left[\log R^{2}\right]\right\rfloor$. Since $Z_{m, n}=Z_{m_{1}, n} \prod_{i=m_{1}+1}^{m} R_{i, n}^{1}$,

$$
N^{-\alpha / 2} \overline{\log Z_{m, n}}=N^{-\alpha / 2} \overline{\log Z_{m_{1}, n}}+N^{-\alpha / 2} \sum_{i=m_{1}+1}^{m} \overline{\log R_{i, n}^{1}}
$$

The sequence $\left(m_{1}, n\right)$ satisfies (1.10). Using Chebyshev's inequality and the upper bound of Theorem 1.2 shows that the term $N^{-\alpha / 2} \overline{\log Z_{m_{1}, n}}$ converges to zero in probability. By the down-right property, the summands in the second term are i.i.d. with mean zero and variance $\operatorname{Var}\left[\log R^{1}\right]$. By the central limit theorem, $N^{-\alpha / 2} \sum_{i=m_{1}+1}^{m} \overline{\log R_{i, n}^{1}}$ converges in distribution to a centered normal with variance $c_{1} \mathbb{V} \operatorname{ar}\left[\log R^{1}\right]$.

\section{Proof of the path fluctuation upper bound}

Given $0 \leqslant k<m$ and $0 \leqslant l<n$, we define a partition function $Z_{m, n}^{(k, l)}$ and quenched polymer measure $Q_{m, n}^{(k, l)}$ on up-right paths from $(k, l)$ to $(m, n)$ by using the collections $\left\{R_{i, l}^{1}: k+1 \leqslant i \leqslant m\right\}$ and $\left\{R_{k, j}^{2}: l+1 \leqslant j \leqslant n\right\}$ as weights along the edges of the south and west boundaries of the rectangle $[k, m] \times[l, n]$ respectively, and the weights $\left\{\left(Y_{z}^{1}, Y_{z}^{2}\right): z \in\{k+1, \ldots, m\} \times\{l+1, \ldots, n\}\right\}$ for the remaining edges. When the original polymer environment (1.1) has the down-right property, it follows that $Z_{m, n}^{(k, l)}$ has the same distribution as $Z_{m-k, n-l}$.

For an up-right path $x$. from $(k, l)$ to $(m, n)$, define

$$
t_{1}^{(k, l)}\left(x_{\bullet}\right):=\max \left\{i:(k+i, l) \in x_{\bullet}\right\}, \quad t_{2}^{(k, l)}\left(x_{\bullet}\right):=\max \left\{j:(k, l+j) \in x_{\bullet}\right\} .
$$

Recall the definition (1.12). 
Lemma 5.1. Assume that the polymer environment satisfies the down-right property. Then for all $0 \leqslant k<m, 0 \leqslant l<n$, and $u \geqslant 0$,

$$
\begin{gathered}
Q_{m, n}\left(v_{1}(l) \geqslant k+u\right)=Q_{m, n}^{(k, l)}\left(t_{1}^{(k, l)} \geqslant u\right) \stackrel{d}{=} Q_{m-k, n-l}\left(t_{1} \geqslant u\right), \\
Q_{m, n}\left(w_{1}(k) \geqslant l+u\right)=Q_{m, n}^{(k, l)}\left(t_{2}^{(k, l)} \geqslant u\right) \stackrel{d}{=} Q_{m-k, n-l}\left(t_{2} \geqslant u\right) .
\end{gathered}
$$

Proof: For $0 \leqslant i<m$ and $0 \leqslant j<n$, we let

$$
Z_{(i, j),(m, n)}:=\sum_{x .} \prod_{k=1}^{(m-i)+(n-j)} \omega_{\left(x_{k-1}, x_{k}\right)}
$$

denote the partition function for up-right paths from $(i, j)$ to $(m, n)$, where the sum is taken over all such paths. A decomposition shows that

$$
\begin{aligned}
Z_{m, n}^{(k, l)}= & \sum_{i=k+1}^{m}\left(\prod_{a=k+1}^{i} R_{a, l}^{1}\right) Y_{i, l+1}^{2} Z_{(i, l+1),(m, n)} \\
& +\sum_{j=l+1}^{n}\left(\prod_{b=l+1}^{j} R_{k, b}^{2}\right) Y_{k+1, j}^{1} Z_{(k+1, j),(m, n)} \\
= & \sum_{i=k+1}^{m} \frac{Z_{i, l}}{Z_{k, l}} Y_{i, l+1}^{2} Z_{(i, l+1),(m, n)}+\sum_{j=l+1}^{n} \frac{Z_{k, j}}{Z_{k, l}} Y_{k+1, j}^{1} Z_{(k+1, j),(m, n)}=\frac{Z_{m, n}}{Z_{k, l}} .
\end{aligned}
$$

We then have that for $r \in\{0, \ldots, m-k\}$,

$$
\begin{aligned}
Q_{m, n}^{(k, l)}\left(t_{1}^{(k, l)}=r\right) & =\frac{1}{Z_{m, n}^{(k, l)}}\left(\prod_{i=1}^{r} R_{k+i, l}^{1}\right) Y_{k+r, l+1}^{2} Z_{(k+r, l+1),(m, n)} \\
& =\frac{1}{Z_{m, n}^{(k, l)}} \frac{Z_{k+r, l}}{Z_{k, l}} Y_{k+r, l+1}^{2} Z_{(k+r, l+1),(m, n)} \\
& =\frac{Z_{k+r, l} Y_{k+r, l+1}^{2} Z_{(k+r, l+1),(m, n)}}{Z_{m, n}} \\
& =Q_{m, n}\left(v_{1}(l)=k+r\right)
\end{aligned}
$$

Summing over $r \geqslant u$ gives the first equality in (5.1). The equality in distribution follows from the down-right property. An analogous argument gives (5.2).

We can now prove the upper bound on the polymer path fluctuations under the annealed measure.

Proof of Theorem 1.5: By assumption, the polymer environment is distributed as in (3.2). If $\tau=0$ this reduces to Lemma 4.7. If $\tau \in(0,1)$ put $(k, l)=(\lfloor\tau m\rfloor,\lfloor\tau n\rfloor)$. By part (c) of Remark 3.2, $\operatorname{Var}\left[R^{i}\right]=\psi_{1}^{f^{i}}\left(a_{i}\right)$ for $i=1,2$. Multiplying (1.10) by $(1-\tau)$, up to integer corrections the sequence $(m-k, n-l)$ satisfies

$$
\left|m-k-M \psi_{1}^{f^{2}}\left(a_{2}\right)\right| \vee\left|n-l-M \psi_{1}^{f^{1}}\left(a_{1}\right)\right| \leqslant \gamma_{0} M^{2 / 3},
$$

where $M=(1-\tau) N$ and $\gamma_{0}=\gamma(1-\tau)^{1 / 3}$. We then apply Lemma 5.1 to get

$$
\begin{aligned}
Q_{m, n}\left(v_{1}(\lfloor\tau n\rfloor) \geqslant \tau m+b N^{2 / 3}\right) & \leqslant Q_{m, n}\left(v_{1}(\lfloor\tau n\rfloor) \geqslant\lfloor\tau m\rfloor+b N^{2 / 3}\right) \\
& \stackrel{d}{=} Q_{m-k, n-l}\left(t_{1} \geqslant b N^{2 / 3}\right) .
\end{aligned}
$$


Applying Lemma 4.7, we get

$$
P_{m, n}\left(v_{1}(\lfloor\tau n\rfloor) \geqslant \tau m+b N^{2 / 3}\right) \leqslant \frac{C}{b^{3}} .
$$

The same argument in the vertical direction gives us

$$
P_{m, n}\left(w_{1}(\lfloor\tau m\rfloor) \geqslant \tau n+b N^{2 / 3}\right) \leqslant \frac{C}{b^{3}} .
$$

To prove the corresponding bounds for $v_{0}$ and $w_{0}$ we now let $k=\left\lfloor\tau m-b N^{2 / 3}\right\rfloor$ and $l=\left\lfloor\tau n-b N^{2 / 3} \frac{n}{m}\right\rfloor$. Again $(m-k, n-l)$ will satisfy (5.3) for a different constant $\gamma_{0}$. Since $w_{1}(k) \geqslant\lfloor\tau n\rfloor$ implies that $v_{0}(\lfloor\tau n\rfloor) \leqslant k$, it follows that

$$
\begin{aligned}
Q_{m, n}\left(v_{0}(\lfloor\tau n\rfloor) \leqslant \tau m-b N^{2 / 3}\right) & \leqslant Q_{m, n}\left(w_{1}(k) \geqslant\lfloor\tau n\rfloor\right) \\
& =Q_{m, n}^{(k, l)}\left(t_{2}^{(k, l)} \geqslant\lfloor\tau n\rfloor-l\right) \\
& \leqslant Q_{m, n}^{(k, l)}\left(t_{2}^{(k, l)} \geqslant C b N^{2 / 3}\right) \\
& \stackrel{d}{=} Q_{m-k, n-l}\left(t_{2} \geqslant C b N^{2 / 3}\right),
\end{aligned}
$$

for some constant $C$ depending on $\left(a_{1}, a_{2}\right), \beta$, and $\gamma$. Applying Lemma 4.7 gives

$$
P_{m, n}\left(v_{0}(\lfloor\tau n\rfloor) \leqslant \tau m-b N^{2 / 3}\right) \leqslant \frac{C}{b^{3}} .
$$

An analogous argument shows that

$$
P_{m, n}\left(w_{0}(\lfloor\tau m\rfloor) \leqslant \tau n-b N^{2 / 3}\right) \leqslant \frac{C}{b^{3}} .
$$

Combining bounds (5.4) and (5.6) gives (1.13), and (5.5) with (5.7) gives (1.14), completing the proof.

\section{Proof of the variance and path fluctuation lower bounds}

Proposition 6.1. Assume that the polymer environment is distributed as in (3.2) and the sequence $(m, n)=\left(m_{N}, n_{N}\right)_{N=1}^{\infty}$ satisfies (1.10) for some positive constant $\gamma$. Then there exist positive constants $c_{0}, \epsilon_{0}, N_{0}$ depending only on $\left(a_{1}, a_{2}\right), \beta$ and $\gamma$ such that for all $N \geqslant N_{0}$,

$$
\mathbb{P}\left(\overline{\log Z_{m, n}} \geqslant c_{0} N^{1 / 3}\right) \geqslant \epsilon_{0} .
$$

From this proposition we can obtain the lower bound of Theorem 1.2:

$$
\begin{aligned}
\operatorname{Var}\left[\log Z_{m, n}\right] & \left.\geqslant \mathbb{E}\left[\overline{\left(\overline{\log Z_{m, n}}\right.}\right)^{2}: \overline{\log Z_{m, n}} \geqslant c_{0} N^{1 / 3}\right] \\
& \geqslant \mathbb{P}\left(\overline{\log Z_{m, n}} \geqslant c_{0} N^{1 / 3}\right)\left(c_{0} N^{1 / 3}\right)^{2} \\
& \geqslant \epsilon_{0} c_{0}^{2} N^{2 / 3} .
\end{aligned}
$$

Proof of Proposition 6.1: Let $\epsilon>0$ be small enough such that for all $|\lambda| \leqslant \epsilon$, $a_{1}+\lambda \in D\left(M_{f^{1}}\right)$ and $a_{2}-\lambda \in D\left(M_{f^{2}}\right)$. Define

$$
\tilde{m}=\left\lfloor m \frac{\psi_{1}^{f^{2}}\left(a_{2}-\lambda\right)}{\psi_{1}^{f^{2}}\left(a_{2}\right)}\right\rfloor, \quad \tilde{n}=\left\lfloor n \frac{\psi_{1}^{f^{1}}\left(a_{1}+\lambda\right)}{\psi_{1}^{f^{1}}\left(a_{1}\right)}\right\rfloor .
$$




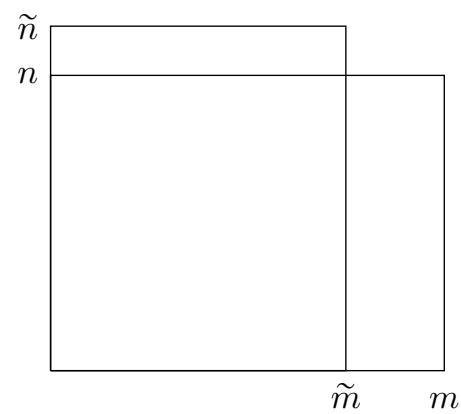

Figure 6.7. Case 1: $\psi_{2}^{f_{1}}$ and $\psi_{2}^{f_{2}}$ are both positive.

Taking Taylor expansions gives

$$
\begin{gathered}
m-\tilde{m}=\lambda \frac{\psi_{2}^{f^{2}}\left(a_{2}\right)}{\psi_{1}^{f^{2}}\left(a_{2}\right)} m+o(\lambda) m \\
\tilde{n}-n=\lambda \frac{\psi_{2}^{f^{1}}\left(a_{1}\right)}{\psi_{1}^{f^{1}}\left(a_{1}\right)} n+o(\lambda) n .
\end{gathered}
$$

Let $b$ be a large fixed constant which will be determined through the course of the proof. Then there exists $N_{0} \in \mathbb{N}$ such that for all $N \geqslant N_{0}, b N^{-1 / 3} \leqslant \epsilon$. Then with $\lambda=b N^{-1 / 3}$, the sequence $(\widetilde{m}, \widetilde{n})$ satisfies

$$
\left|\tilde{m}-N \psi_{1}^{f^{2}}\left(a_{2}-\lambda\right)\right| \vee\left|\widetilde{n}-N \psi_{1}^{f^{1}}\left(a_{1}+\lambda\right)\right| \leqslant \gamma_{0} N^{2 / 3}
$$

for some positive constant $\gamma_{0}$. By Table C.9 and (C.1) in the Appendix, in each of the four basic beta-gamma models, either $\psi_{2}^{f^{1}}\left(a_{1}\right)$ and $\psi_{2}^{f^{2}}\left(a_{2}\right)$ are both positive (inverse-beta model for certain choices of parameters and inverse-gamma model for all choices of parameters), $\psi_{2}^{f^{1}}\left(a_{1}\right)$ is negative and $\psi_{2}^{f^{2}}\left(a_{2}\right)$ is positive (gamma and beta models), or $\psi_{2}^{f^{1}}\left(a_{1}\right)$ is positive and $\psi_{2}^{f^{2}}\left(a_{2}\right)$ is non-positive (inverse-beta model with the remaining choices of parameters). By flipping the $x$ and $y$ axes in the second case, we only need to consider the first and third cases.

For the case where $\psi_{2}^{f^{1}}\left(a_{1}\right)$ and $\psi_{2}^{f^{2}}\left(a_{2}\right)$ are both positive define $A_{N}=m-\tilde{m}$ and $B_{N}=\tilde{n}-n$. This case is illustrated in Figure 6.7. By (6.1) and increasing $N_{0}$ if necessary, there exist positive constants $c_{1}, c_{2}, C_{1}, C_{2}$ such that for $N \geqslant N_{0}$,

$$
\begin{aligned}
& c_{1} b N^{2 / 3} \leqslant A_{N} \leqslant C_{1} b N^{2 / 3}, \\
& c_{2} b N^{2 / 3} \leqslant B_{N} \leqslant C_{2} b N^{2 / 3} .
\end{aligned}
$$

In the case where $\psi_{2}^{f^{1}}\left(a_{1}\right)>0$ and $\psi_{2}^{f^{2}}\left(a_{2}\right) \leqslant 0$ we define $c:=\frac{1}{2}\left(\frac{m}{\widetilde{m}}+\frac{n}{\widetilde{n}}\right)$ and let $\bar{m}=c \tilde{m}, \bar{n}=c \tilde{n}$. This case is illustrated in Figure 6.8. This $(\bar{m}, \bar{n})$ will satisfy

$$
\left|\bar{m}-M \psi_{1}^{f^{2}}\left(a_{2}-\lambda\right)\right| \vee\left|\bar{n}-M \psi_{1}^{f^{1}}\left(a_{1}+\lambda\right)\right| \leqslant \gamma_{0} c^{1 / 3} M^{2 / 3}
$$

where $M=c N$. A Taylor expansion gives

$$
c=1+\left(\frac{\psi_{2}^{f^{2}}\left(a_{2}\right)}{\psi_{1}^{f^{2}}\left(a_{2}\right)}-\frac{\psi_{2}^{f^{1}}\left(a_{1}\right)}{\psi_{1}^{f^{1}}\left(a_{1}\right)}\right) \frac{\lambda}{2}+o(\lambda)
$$




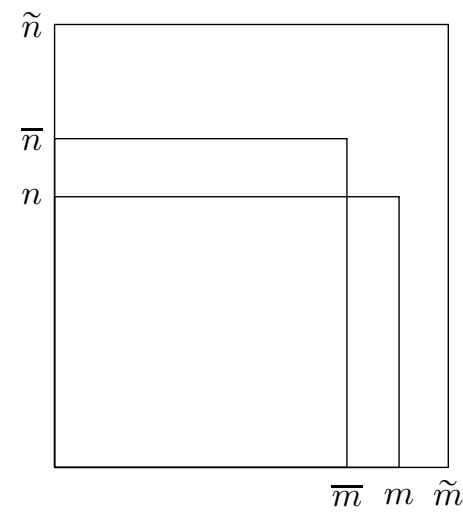

Figure 6.8 . Case $2: \psi_{2}^{f_{1}}>0$ and $\psi_{2}^{f_{2}} \leqslant 0$.

and thus

$$
\begin{gathered}
m-\bar{m}=\frac{\lambda}{2}\left(\frac{\psi_{2}^{f^{2}}\left(a_{2}\right)}{\psi_{1}^{f^{2}}\left(a_{2}\right)}+\frac{\psi_{2}^{f^{1}}\left(a_{1}\right)}{\psi_{1}^{f^{1}}\left(a_{1}\right)}\right) m+o\left(N^{2 / 3}\right), \\
\bar{n}-n=\frac{\lambda}{2}\left(\frac{\psi_{2}^{f^{2}}\left(a_{2}\right)}{\psi_{1}^{f^{2}}\left(a_{2}\right)}+\frac{\psi_{2}^{f^{1}}\left(a_{1}\right)}{\psi_{1}^{f^{1}}\left(a_{1}\right)}\right) n+o\left(N^{2 / 3}\right) .
\end{gathered}
$$

The quantity $\frac{\psi_{2}^{f^{2}}\left(a_{2}\right)}{\psi_{1}^{f^{2}}\left(a_{2}\right)}+\frac{\psi_{2}^{f^{1}}\left(a_{1}\right)}{\psi_{1}^{f^{1}}\left(a_{1}\right)}$ is positive since $\psi_{1}^{f^{1}}$ and $\psi_{1}^{f^{2}}$ are both positive and $\psi_{1}^{f^{2}}\left(a_{2}\right) \psi_{2}^{f^{1}}\left(a_{1}\right)+\psi_{1}^{f^{1}}\left(a_{1}\right) \psi_{2}^{f^{2}}\left(a_{2}\right)>0$ by Lemma C.2 in the Appendix. Letting $\bar{A}=m-\bar{m}$ and $\bar{B}=\bar{n}-n$, there exist positive constants $c_{1}^{\prime}, c_{2}^{\prime}, C_{1}^{\prime}, C_{2}^{\prime}$ such that

$$
\begin{aligned}
& c_{1}^{\prime} b M^{2 / 3} \leqslant \bar{A}_{M} \leqslant C_{1}^{\prime} b M^{2 / 3}, \\
& c_{2}^{\prime} b M^{2 / 3} \leqslant \bar{B}_{M} \leqslant C_{2}^{\prime} b M^{2 / 3} .
\end{aligned}
$$

Recall that $\mathbb{P}^{\left(a_{1}, a_{2}\right)}$ is used to denote the probability measure on the polymer environment with parameters $a_{1}$ and $a_{2}$. Let $\left(\tilde{a}_{1}, \tilde{a}_{2}\right)=\left(a_{1}+\lambda, a_{2}-\lambda\right)$. Our goal is to show that

$$
\mathbb{P}^{\left(a_{1}, a_{2}\right)}\left(\log Z_{m, n} \geqslant \mathbb{E}\left[\log Z_{m, n}\right]+c_{0} N^{1 / 3}\right) \geqslant \epsilon_{0}
$$

We will do so by making estimates using the $\left(\widetilde{a}_{1}, \widetilde{a}_{2}\right)$ environment and then use a coupling of the two environments to transfer the results to the $\left(a_{1}, a_{2}\right)$ environment.

We would first like to show that in the $\left(\tilde{a}_{1}, \tilde{a}_{2}\right)$ environment, with high probability the quenched probability gives most of the weight to paths which exit the $x$-axis at a point of order $b N^{2 / 3}$. That is: there exist positive constants $C_{3}, C$ such that, given any $\varepsilon>0$,

$$
\mathbb{P}^{\left(\widetilde{a}_{1}, \widetilde{a}_{2}\right)}\left\{Q_{m, n}\left(c_{1} b N^{2 / 3} \leqslant t_{1} \leqslant C_{3} b N^{2 / 3}\right) \geqslant 1-\varepsilon\right\} \geqslant 1-\frac{C}{b^{3}}
$$

holds for all sufficiently large $N$.

We start by using Lemma 5.1 to relate an upper bound on $t_{1}$ to a lower bound on $t_{2}$.

$$
Q_{m, n}\left(t_{1} \leqslant A_{N}\right) \stackrel{d}{=} Q_{m, \widetilde{n}}\left(v_{1}\left(B_{N}\right) \leqslant A_{N}\right)=Q_{m, \widetilde{n}}\left(w_{1}\left(A_{N}\right)>B_{N}\right) \stackrel{d}{=} Q_{\widetilde{m}, \widetilde{n}}\left(t_{2}>B_{N}\right) .
$$


Using this and Corollary 4.6, there exists $\delta>0$ such that

$$
\begin{aligned}
\mathbb{P}^{\left(\widetilde{a}_{1}, \tilde{a}_{2}\right)}\left\{Q_{m, n}\left(t_{1}>c_{1} b N^{2 / 3}\right) \geqslant 1\right. & \left.-e^{-\frac{\delta}{N} B_{N}^{2}}\right\} \\
& \geqslant \mathbb{P}^{\left(\widetilde{a}_{1}, \widetilde{a}_{2}\right)}\left\{Q_{m, n}\left(t_{1}>A_{N}\right) \geqslant 1-e^{-\frac{\delta}{N} B_{N}^{2}}\right\} \\
& =\mathbb{P}^{\left(\widetilde{a}_{1}, \widetilde{a}_{2}\right)}\left\{Q_{m, n}\left(t_{1} \leqslant A_{N}\right) \leqslant e^{-\frac{\delta}{N} B_{N}^{2}}\right\} \\
& =\mathbb{P}^{\left(\widetilde{a}_{1}, \widetilde{a}_{2}\right)}\left\{Q_{\widetilde{m}, \tilde{n}}\left(t_{2}>B_{N}\right) \leqslant e^{-\frac{\delta}{N} B_{N}^{2}}\right\} \\
& \geqslant 1-C b^{-3} .
\end{aligned}
$$

This implies that

$$
\mathbb{P}^{\left(\widetilde{a}_{1}, \widetilde{a}_{2}\right)}\left\{Q_{m, n}\left(t_{1} \leqslant c_{1} b N^{2 / 3}\right) \geqslant e^{-\frac{\delta}{N} B_{N}^{2}}\right\} \leqslant C b^{-3} .
$$

Applying the upper bound (4.17) directly with $C_{3}>C_{2}$, we obtain

$$
\mathbb{P}^{\left(\widetilde{a}_{1}, \widetilde{a}_{2}\right)}\left\{Q_{m, n}\left(t_{1}>C_{3} b N^{2 / 3}\right) \geqslant e^{-\frac{\delta}{N} B_{N}^{2}}\right\} \leqslant C b^{-3}
$$

for another positive constant $C$. Taking a union bound we put the two bounds together and get

$$
\mathbb{P}^{\left(\widetilde{a}_{1}, \tilde{a}_{2}\right)}\left\{Q_{m, n}\left(c_{1} b N^{2 / 3} \leqslant t_{1} \leqslant C_{3} b N^{2 / 3}\right) \geqslant 1-2 e^{-\frac{\delta}{N} B_{N}^{2}}\right\} \geqslant 1-C b^{-3} .
$$

Taking $N$ large enough, we get (6.2).

The argument for the case where we use $(\bar{m}, \bar{n})$ and $\bar{A}, \bar{B}$ is unchanged, with the exception of using the scaling parameter $M$ rather than $N$. This difference can be absorbed into the constants.

In order to make use of the bound (6.2) for the system with the original $\left(a_{1}, a_{2}\right)$ environment we create a new measure $\breve{\mathbb{P}}$ which has both $a_{1}$ and $\tilde{a}_{1}$ distributed weights along the $x$-axis and estimate the Radon-Nikodym derivative of the $\left(a_{1}, a_{2}\right)$ environment with respect to this new environment.

Let $\widetilde{\omega}$ denote the environment that has the same weights as the $\left(a_{1}, a_{2}\right)$ environment except for the weights $R_{i, 0}^{1}$ for $1 \leqslant i \leqslant\left\lfloor C_{3} b N^{2 / 3}\right\rfloor$, which will be distributed with parameter $\tilde{a}_{1}$. Let $\breve{\mathbb{P}}$ denote the probability measure of this environment. Then for each path $x$. with $c_{1} b N^{2 / 3} \leqslant t_{1}(x) \leqslant C_{3} b N^{2 / 3}$, the weight of the path in the $\left(\widetilde{a}_{1}, \tilde{a}_{2}\right)$ environment and the weight of the path in the $\check{\omega}$ environment agree. Thus, defining $Z_{m, n}(A):=\sum_{x, \in A} \prod_{k=1}^{m+n} \omega_{\left(x_{k-1}, x_{k}\right)}$,

$$
Z_{m, n}\left(c_{1} b N^{2 / 3} \leqslant t_{1} \leqslant C_{3} b N^{2 / 3}\right)
$$

is the same in distribution under $\mathbb{P}^{\left(\widetilde{a}_{1}, \widetilde{a}_{2}\right)}$ and $\breve{\mathbb{P}}$. We can now make use of the bound (6.2).

Using a third-order Taylor expansion, the same series of calculations which leads to inequality (4.11) in the proof of Proposition 4.3 gives the existence of a constant $C^{\prime}>0$ such that:

$$
\begin{aligned}
\mathbb{E}^{\left(\widetilde{a}_{1}, \widetilde{a}_{2}\right)}\left[\log Z_{m, n}\right]-\mathbb{E}^{\left(a_{1}, a_{2}\right)}\left[\log Z_{m, n}\right]= & m\left(\Psi_{0}^{f_{1}}\left(\widetilde{a}_{1}\right)-\Psi_{0}^{f_{1}}\left(a_{1}\right)\right) \\
& +n\left(\Psi_{0}^{f_{2}}\left(\widetilde{a}_{2}\right)-\Psi_{0}^{f_{2}}\left(a_{2}\right)\right) \\
\geqslant & -\gamma b N^{1 / 3} C^{\prime}+4 c_{4} b^{2} N^{1 / 3}-b^{3} C^{\prime} \\
& \geqslant c_{4} b^{2} N^{1 / 3}
\end{aligned}
$$


where $c_{4}:=\frac{1}{8}\left(\psi_{1}^{f^{2}}\left(a_{2}\right) \psi_{2}^{f^{1}}\left(a_{1}\right)+\psi_{1}^{f^{1}}\left(a_{1}\right) \psi_{2}^{f^{2}}\left(a_{2}\right)\right)$ is positive by Lemma C.2 in the Appendix. The last inequality is obtained by first fixing $b$ large enough then increasing $N_{0}$ if necessary.

We now split the probability

$$
\begin{aligned}
& \mathbb{P}^{\left(\widetilde{a}_{1}, \tilde{a}_{2}\right)}\left\{Q_{m, n}\left(c_{1} b N^{2 / 3} \leqslant t_{1} \leqslant C_{3} b N^{2 / 3}\right) \geqslant 1-\varepsilon\right\} \\
& =\mathbb{P}^{\left(\widetilde{a}_{1}, \widetilde{a}_{2}\right)}\left\{\frac{1}{Z_{m, n}} Z_{m, n}\left(c_{1} b N^{2 / 3} \leqslant t_{1} \leqslant C_{3} b N^{2 / 3}\right) \geqslant 1-\varepsilon\right\} \\
& \leqslant \check{\mathbb{P}}\left\{Z_{m, n}\left(c_{1} b N^{2 / 3} \leqslant t_{1} \leqslant C_{3} b N^{2 / 3}\right) \geqslant(1-\varepsilon) e^{\mathbb{E}^{\left(\tilde{a}_{1}, \tilde{a}_{2}\right)}\left[\log Z_{m, n}\right]-\frac{1}{2} c_{4} b^{2} N^{1 / 3}}\right\} \\
& +\mathbb{P}^{\left(\widetilde{a}_{1}, \widetilde{a}_{2}\right)}\left\{Z_{m, n} \leqslant e^{\mathbb{E}^{\left(\tilde{a}_{1}, \tilde{a}_{2}\right)}\left[\log Z_{m, n}\right]-\frac{1}{2} c_{4} b^{2} N^{1 / 3}}\right\} \\
& \leqslant \check{\mathbb{P}}\left\{Z_{m, n}\left(c_{1} b N^{2 / 3} \leqslant t_{1} \leqslant C_{3} b N^{2 / 3}\right) \geqslant(1-\varepsilon) e^{\mathbb{E}^{\left(a_{1}, a_{2}\right)}\left[\log Z_{m, n}\right]+\frac{1}{2} c_{4} b^{2} N^{1 / 3}}\right\} \\
& +\mathbb{P}^{\left(\widetilde{a}_{1}, \widetilde{a}_{2}\right)}\left\{Z_{m, n} \leqslant e^{\mathbb{E}^{\left(\widetilde{a}_{1}, \widetilde{a}_{2}\right)}\left[\log Z_{m, n}\right]-\frac{1}{2} c_{4} b^{2} N^{1 / 3}}\right\} .
\end{aligned}
$$

The transition from $\mathbb{P}^{\left(\widetilde{a}_{1}, \widetilde{a}_{2}\right)}$ to $\breve{\mathbb{P}}$ in $(6.5)$ is due to the equality in distribution of (6.3) under these measures. Inequality (6.6) comes from (6.4).

For (6.7) we can use Chebyshev's inequality then the upper bound of the variance to get

$$
(6.7) \leqslant \frac{C}{b^{3}}
$$

Thus $(6.6) \geqslant 1-\frac{C}{b^{3}}$ for some new positive constant $C$. Let $g$ be the RadonNikodym derivative $d \breve{\mathbb{P}} / d \mathbb{P}^{\left(a_{1}, a_{2}\right)}$. Recall that the distributions differ only on the weights along the $x$-axis up until site $\left[C_{3} b N^{2 / 3}\right\rfloor$. Thus

$$
g(\omega)=\left(\frac{M_{f^{1}}\left(a_{1}\right)}{M_{f^{1}}\left(\widetilde{a}_{1}\right)}\right)^{\left\lfloor C_{3} b N^{2 / 3}\right\rfloor\left\lfloor C_{3} b N^{2 / 3}\right\rfloor} \prod_{i=1}^{\lambda} \omega_{i, 0}^{\lambda} .
$$

We can evaluate $\mathbb{E}^{\left(a_{1}, a_{2}\right)}\left[g^{2}\right]$ explicitly. Increasing $N_{0}$, if necessary, so that $2 \lambda \leqslant \epsilon$,

$$
\mathbb{E}^{\left(a_{1}, a_{2}\right)}\left[\omega_{i, 0}^{2 \lambda}\right]=\frac{1}{M_{f^{1}}\left(a_{1}\right)} \int_{0}^{\infty} x^{2 \lambda} x^{a_{1}-1} f^{1}(x) d x=\frac{M_{f^{1}}\left(a_{1}+2 \lambda\right)}{M_{f^{1}}\left(a_{1}\right)} .
$$

Now

$$
\begin{aligned}
\mathbb{E}^{\left(a_{1}, a_{2}\right)}\left[g^{2}\right] & =\left(\frac{M_{f^{1}}\left(a_{1}\right)}{M_{f^{1}}\left(\widetilde{a}_{1}\right)}\right)^{2\left\lfloor C_{3} b N^{2 / 3}\right\rfloor} \prod_{i=1}^{\left\lfloor C_{3} b N^{2 / 3}\right\rfloor} \mathbb{E}^{\left(a_{1}, a_{2}\right)}\left[\omega_{i, 0}^{2 \lambda}\right] \\
& =\left(\frac{M_{f^{1}}\left(a_{1}\right) M_{f^{1}}\left(a_{1}+2 \lambda\right)}{M_{f^{1}}\left(a_{1}+\lambda\right)^{2}}\right)^{\left\lfloor C_{3} b N^{2 / 3}\right\rfloor} .
\end{aligned}
$$

Taking logarithms of both sides,

$$
\begin{aligned}
\log \mathbb{E}^{\left(a_{1}, a_{2}\right)}\left[g^{2}\right] \\
\quad=\left\lfloor C_{3} b N^{2 / 3}\right\rfloor\left(\log M_{f^{1}}\left(a_{1}\right)+\log M_{f^{1}}\left(a_{1}+2 b N^{-1 / 3}\right)-2 \log M_{f^{1}}\left(a_{1}+b N^{-1 / 3}\right)\right)
\end{aligned}
$$


Recall that $\frac{\partial^{2}}{\partial a^{2}} \log M_{f^{1}}(a)=\psi_{1}^{f^{1}}(a)>0$. Then

$$
\begin{aligned}
\lim _{N \rightarrow \infty} & \log \mathbb{E}^{\left(a_{1}, a_{2}\right)}\left[g^{2}\right] \\
& =C_{3} b \lim _{N \rightarrow \infty} \frac{\log M_{f^{1}}\left(a_{1}\right)+\log M_{f^{1}}\left(a_{1}+2 b N^{-1 / 3}\right)-2 \log M_{f^{1}}\left(a_{1}+b N^{-1 / 3}\right)}{N^{-2 / 3}} \\
& =C_{3} b^{2} \lim _{N \rightarrow \infty} \frac{\psi_{0}^{f^{1}}\left(a_{1}+2 b N^{-1 / 3}\right)-\psi_{0}^{f^{1}}\left(a_{1}+b N^{-1 / 3}\right)}{N^{-1 / 3}} \\
& =C_{3} b^{3} \psi_{1}^{f^{1}}\left(a_{1}\right)>0
\end{aligned}
$$

Increase $N_{0}$ if necessary so that for all $N \geqslant N_{0}$,

$$
\mathbb{E}^{\left(a_{1}, a_{2}\right)}\left[g^{2}\right] \leqslant e^{2 C_{3} b^{3}}
$$

Defining the event

$$
D=\left\{Z_{m, n}\left(c_{1} b N^{2 / 3} \leqslant t_{1} \leqslant C_{3} b N^{2 / 3}\right) \geqslant(1-\epsilon) e^{\mathbb{E}^{\left(a_{1}, a_{2}\right)}\left[\log Z_{m, n}\right]+\frac{1}{2} c_{4} b^{2} N^{1 / 3}}\right\},
$$

we get

$$
\begin{aligned}
1-\frac{C}{b^{3}} \leqslant(6.6) & =\breve{\mathbb{P}}(D) \\
& =\mathbb{E}^{\left(a_{1}, a_{2}\right)}\left[g \mathbb{1}_{D}\right] \\
& \leqslant\left(\mathbb{E}^{\left(a_{1}, a_{2}\right)}\left[g^{2}\right]\right)^{1 / 2}\left(\mathbb{P}^{\left(a_{1}, a_{2}\right)}(D)\right)^{1 / 2} \\
& \leqslant e^{C_{3} b^{3}}\left(\mathbb{P}^{\left(a_{1}, a_{2}\right)}(D)\right)^{1 / 2} .
\end{aligned}
$$

Thus

$$
\epsilon_{0}:=\left(1-\frac{C}{b^{3}}\right)^{2} e^{-2 C_{3} b^{3}} \leqslant \mathbb{P}^{\left(a_{1}, a_{2}\right)}(D)
$$

Finally we have that

$$
\begin{aligned}
\epsilon_{0} \leqslant \mathbb{P}^{\left(a_{1}, a_{2}\right)}(D) & \leqslant \mathbb{P}^{\left(a_{1}, a_{2}\right)}\left(Z_{m, n} \geqslant(1-\varepsilon) e^{\mathbb{E}^{\left(a_{1}, a_{2}\right)}\left[\log Z_{m, n}\right]+\frac{1}{2} c_{4} b^{2} N^{1 / 3}}\right) \\
& =\mathbb{P}^{\left(a_{1}, a_{2}\right)}\left(\log Z_{m, n} \geqslant \log (1-\varepsilon)+\mathbb{E}^{\left(a_{1}, a_{2}\right)}\left[\log Z_{m, n}\right]+\frac{c_{4} b^{2} N^{1 / 3}}{2}\right) \\
& \leqslant \mathbb{P}^{\left(a_{1}, a_{2}\right)}\left(\log Z_{m, n} \geqslant \mathbb{E}^{\left(a_{1}, a_{2}\right)}\left[\log Z_{m, n}\right]+c_{0} N^{1 / 3}\right) .
\end{aligned}
$$

Increasing $N_{0}$ if necessary and taking $c_{0}=\frac{1}{4} c_{4} b^{2}$ the final inequality holds for all $N \geqslant N_{0}$. This concludes the proof.

We can use the variance lower bound to obtain a lower bound on the exit points of the path from the horizontal and vertical axes.

Corollary 6.2. Assume that the polymer environment is distributed as in (3.2) and the sequence $(m, n)=\left(m_{N}, n_{N}\right)_{N=1}^{\infty}$ satisfies (1.10) for some positive constant $\gamma$. Then there exist positive constants $c_{0}, c_{1}, N_{0}$ depending only on $\left(a_{1}, a_{2}\right), \beta$ and $\gamma$ such that for all $N \geqslant N_{0}$,

$$
c_{0} \leqslant P_{m, n}\left(t_{1}>c_{1} N^{2 / 3} \text { or } t_{2}>c_{1} N^{2 / 3}\right) .
$$


Proof: Averaging (1.8) and (1.9) of Proposition 1.1 then applying Lemma 4.2 followed by the lower bound of Theorem 1.2 gives the existence of positive constants c, $C, N_{0}$ such that for all $N \geqslant N_{0}$

$$
\begin{aligned}
c N^{2 / 3} \leqslant \operatorname{Var}\left[\log Z_{m, n}\right] & =E_{m, n}\left[\sum_{i=1}^{t_{1}} L_{R^{1}}\left(R_{i, 0}^{1}\right)\right]+E_{m, n}\left[\sum_{j=1}^{t_{2}} L_{R^{2}}\left(R_{0, j}^{2}\right)\right] \\
& \leqslant C\left(E_{m, n}\left[t_{1}+t_{2}\right]+2\right) .
\end{aligned}
$$

Letting $c_{1}:=c / 6 C$ and increasing $N_{0}$ if necessary followed by an application of the Cauchy-Schwartz inequality along with Lemma 4.7 gives

$$
\begin{aligned}
3 c_{1} \leqslant E_{m, n}\left[\frac{t_{1}+t_{2}}{N^{2 / 3}}\right] & \leqslant 2 c_{1}+E_{m, n}\left[\frac{t_{1}+t_{2}}{N^{2 / 3}}: t_{1}+t_{2}>2 c_{1} N^{2 / 3}\right] \\
& \leqslant 2 c_{1}+C^{\prime} P_{m, n}\left(t_{1}+t_{2}>2 c_{1} N^{2 / 3}\right)^{\frac{1}{2}}
\end{aligned}
$$

for some positive constant $C^{\prime}$. Thus

$$
c_{0}:=\left(\frac{c_{1}}{C^{\prime}}\right)^{2} \leqslant P_{m, n}\left(t_{1}+t_{2}>2 c_{1} N^{2 / 3}\right) \leqslant P_{m, n}\left(t_{1}>c_{1} N^{2 / 3} \text { or } t_{2}>c_{1} N^{2 / 3}\right),
$$

which completes the proof.

We now prove the path fluctuation lower bound.

Proof of (1.15): If $\tau=0$, this reduces to Corollary 6.2. If $\tau \in(0,1)$ put $(k, l)=$ $(\lfloor\tau m\rfloor,\lfloor\tau n\rfloor)$. Then the sequence $(m-k, n-l)$ satisfies $(1.10)$ with a new scaling parameter $M=(1-\tau) N$. By the down-right property and Lemma 5.1

$$
\begin{aligned}
Q_{m-k, n-l}\left(t_{1}>u \text { or } t_{2}>u\right) & \stackrel{d}{=} Q_{m, n}^{(k, l)}\left(t_{1}^{(k, l)}>u \text { or } t_{2}^{(k, l)}>u\right) \\
& =Q_{m, n}\left(v_{1}(l)>k+u \text { or } w_{1}(k)>l+u\right) \\
& \leqslant Q_{m, n}\left(v_{1}(l)>\tau m+\frac{u}{2} \text { or } w_{1}(k)>\tau n+\frac{u}{2}\right)
\end{aligned}
$$

provided that $u \geqslant 2$. Corollary 6.2 applied to the sequence $(m-k, n-l)$ completes the proof.

\section{Appendix Appendix A Verification of Hypothesis 3.6}

Lemma A.1. If the function $f$ satisfies the conditions of Hypothesis 3.6 and $g(x):=f\left(\frac{1}{x}\right)$ for $x \in(0, \infty)$, then $g$ also satisfies the conditions of Hypothesis 3.6.

Proof: Note that $\operatorname{supp}(g)=\operatorname{supp}(f)^{-1}$. Fix a compact $K \subset D\left(M_{g}\right)$ and let $a \in K$. By parts (c) and (b) of Remark 3.3, $\psi_{0}^{g}(a)=-\psi_{0}^{f}(-a)$ and $-K \subset D\left(M_{f}\right)$. Thus there exists a positive constant $C$ depending only $-K$ such that for all $b \in-K$, (3.9) and (3.10) hold. It therefore suffices to show the following two relations hold:

$$
\begin{aligned}
L^{g}(a, x) & =L^{f}\left(-a, \frac{1}{x}\right) \quad \text { for all } x \in \operatorname{supp}(g) \\
\int_{0}^{1}\left|\frac{\partial}{\partial a} L^{g}\left(a, H^{g}(a, p)\right)\right| d p & =\int_{0}^{1}\left|\frac{\partial}{\partial b} L^{f}\left(b, H^{f}(b, p)\right)\right| d p
\end{aligned}
$$

where the right hand side of (A.2) is evaluated at $b=-a$.

(A.1) can be proven by using $\psi_{0}^{g}(a)=-\psi_{0}^{f}(-a)$ and making the substitution $y \mapsto \frac{1}{y}$ in the first integral appearing in (3.8). 
(A.2) will now follow from (A.1) and

$$
H^{g}(a, 1-p)=\frac{1}{H^{f}(-a, p)} \quad \text { for all } p \in(0,1)
$$

To see that this equality holds, let $X \sim m_{g}(a)$ and $x>0$. Using part (a) of Remark 3.3

$$
F^{g}(a, x)=\mathbb{P}(X \leqslant x)=\mathbb{P}\left(X^{-1} \geqslant x^{-1}\right)=1-\mathbb{P}\left(X^{-1}<x^{-1}\right)=1-F^{f}\left(-a, x^{-1}\right) .
$$

Fix $p \in(0,1)$ and recall the definition of $H^{\bullet},(3.3)$. Note that $H^{f}(-a, p)$ and $H^{g}(a, 1-p)$ lie in $\operatorname{supp}(f)$ and $\operatorname{supp}(g)=\operatorname{supp}(f)^{-1}$ respectively. Plugging $x=$ $H^{g}(a, 1-p)$ into (A.3) gives

$$
1-p=F^{g}\left(a, H^{g}(a, 1-p)\right)=1-F^{f}\left(-a, \frac{1}{H^{g}(a, 1-p)}\right) .
$$

Rearranging yields

$$
F^{f}\left(-a, \frac{1}{H^{g}(a, 1-p)}\right)=p=F^{f}\left(-a, H^{f}(-a, p)\right) .
$$

Since $x \mapsto F^{f}(-a, x)$ is one-to-one on $\operatorname{supp}(f)$ we have the desired result.

Lemma A.2. Each of the functions $f$ in Table 3.5 satisfy Hypothesis 3.6.

Proof: Fix $b>0$. By Lemma A.1 it suffices to show the three functions

$$
f(x)=e^{-b x}, \quad f(x)=(1-x)^{b-1} \mathbb{1}_{\{0<x<1\}}, \quad f(x)=\left(\frac{x}{x+1}\right)^{b}
$$

satisfy the conditions of Hypothesis 3.6. In Seppäläinen (2012) (equation 3.30 and the computation following equation 4.7), Seppäläinen showed that the function $f(x)=e^{-x}$ satisfies these conditions. A simple rescaling then shows that these conditions are also satisfied for $f(x)=e^{-b x}$.

We will write $C_{0}(a), C_{1}(a), \ldots$ to indicate the positive constants $C_{k}(a)$ have a continuous dependence on $a$. We claim it is sufficient to show that if $f(x)=$ $(1-x)^{b-1} \mathbb{1}_{\{0<x<1\}}$ or $f(x)=\left(\frac{x}{x+1}\right)^{b}$, then for all $x \in \operatorname{supp}(f)$ the following three bounds hold:

$$
\begin{aligned}
L^{f}(a, x) & \leqslant C_{0}(a)(1+|\log x|) \\
\left|x \frac{f^{\prime}(x)}{f(x)}\right| L^{f}(a, x) & \leqslant C_{1}(a)(1+|\log x|) \\
\left|G^{f}(a, x)\right| & \leqslant C_{2}(a)\left(1+(\log x)^{2}\right)
\end{aligned}
$$

where

$$
\begin{aligned}
G^{f}(a, x): & =\frac{x^{-a}}{f(x)} \int_{0}^{x}\left(\psi_{1}^{f}(a)+\psi_{0}^{f}(a) \log y-(\log y)^{2}\right) y^{a-1} f(y) d y \\
& =-\frac{x^{-a}}{f(x)} \int_{x}^{\infty}\left(\psi_{1}^{f}(a)+\psi_{0}^{f}(a) \log y-(\log y)^{2}\right) y^{a-1} f(y) d y .
\end{aligned}
$$

Note that the second equality in the definition of $G^{f}(a, x)$ follows from the definitions of $\psi_{0}^{f}(a)$ and $\psi_{1}^{f}(a)$ in part (c) of Remark 3.2. (A.4) clearly implies (3.9). To 
show (3.10) is satisfied, using (3.7), we calculate

$$
\begin{aligned}
\frac{\partial}{\partial a} L^{f}\left(a, H^{f}(a, p)\right) & =\frac{\partial L^{f}}{\partial a}\left(a, H^{f}(a, p)\right)+\frac{\partial}{\partial a} H^{f}(a, p) \frac{\partial L^{f}}{\partial x}\left(a, H^{f}(a, p)\right) \\
& =\left.\left(\frac{\partial L^{f}}{\partial a}(a, x)+x L^{f}(a, x) \frac{\partial L^{f}}{\partial x}(a, x)\right)\right|_{x=H^{f}(a, p)} .
\end{aligned}
$$

Since

$$
\begin{aligned}
\frac{\partial L^{f}}{\partial a}(a, x)+x L^{f}(a, x) \frac{\partial L^{f}}{\partial x}(a, x)= & \left(\psi_{0}^{f}(a)-2 \log x\right) L^{f}(a, x)-a L^{f}(a, x)^{2} \\
& +G^{f}(a, x)-x \frac{f^{\prime}(x)}{f(x)} L^{f}(a, x)^{2},
\end{aligned}
$$

the conditions (A.4), (A.5), and (A.6) imply the existence of a positive constant $C_{3}(a)$ such that for all $x \in \operatorname{supp}(f)$,

$$
\left|\frac{\partial L^{f}}{\partial a}(a, x)+x L^{f}(a, x) \frac{\partial L^{f}}{\partial x}(a, x)\right| \leqslant C_{3}(a)\left(1+(\log x)^{2}\right) .
$$

Condition (3.10) now follows from

$$
\begin{aligned}
\int_{0}^{1}\left|\frac{\partial}{\partial a} L^{f}\left(a, H^{f}(a, p)\right)\right| d p & \leqslant C_{3}(a) \int_{0}^{1}\left(1+\left(\log H^{f}(a, p)\right)^{2}\right) d p \\
& =C_{3}(a)\left(1+\psi_{1}^{f}(a)+\left(\psi_{0}^{f}(a)\right)^{2}\right)<\infty
\end{aligned}
$$

The last equality is justified by parts (a) and (c) of Remark 3.2 along with the fact that $H^{f}(a, \eta) \sim m_{f}(a)$ when $\eta$ is uniformly distributed on $(0,1)$.

We first show (A.4), (A.5) and (A.6) for the case $f(x)=(1-x)^{b-1} \mathbb{1}_{\{0<x<1\}}$. Let $a \in D\left(M_{f}\right)=(0, \infty)$. Then there exists some positive constant $C_{4}(a)$ such that the following two inequalities hold:

$$
\begin{array}{r}
\left|\psi_{0}^{f}(a)-\log y\right| y^{a-1} f(y) \leqslant \begin{cases}C_{4}(a)(1-\log y) y^{a-1} & \text { if } 0<y<\frac{1}{2} \\
C_{4}(a)(1-y)^{b-1} & \text { if } \frac{1}{2} \leqslant y<1\end{cases} \\
\left|\psi_{1}^{f}(a)+\psi_{0}^{f}(a) \log y-(\log y)^{2}\right| y^{a-1} f(y) \leqslant \begin{cases}C_{4}(a)\left(1+(\log y)^{2}\right) y^{a-1} & \text { if } 0<y<\frac{1}{2} \\
C_{4}(a)(1-y)^{b-1} & \text { if } \frac{1}{2} \leqslant y<1 .\end{cases}
\end{array}
$$

Since $a>0,(3.8)$ and (A.7) give: for $0<x<\frac{1}{2}$,

$$
\begin{gathered}
L^{f}(a, x) \leqslant \frac{2^{b} C_{4}(a)}{x^{a}} \int_{0}^{x}(1-\log y) y^{a-1} d y \leqslant C_{0}(a)(1+|\log x|) \\
\left|G^{f}(a, x)\right| \leqslant \frac{2^{b} C_{4}(a)}{x^{a}} \int_{0}^{x}\left(1+(\log y)^{2}\right) y^{a-1} d y \leqslant C_{2}(a)\left(1+(\log x)^{2}\right) .
\end{gathered}
$$

Similarly, the secondary expressions in (3.8) and (A.7) give: for $1 / 2 \leqslant x<1$,

$$
\begin{array}{r}
L^{f}(a, x) \leqslant \frac{2^{a} C_{4}(a)}{(1-x)^{b-1}} \int_{x}^{1}(1-y)^{b-1} d y \leqslant C_{0}(a)(1-x) \\
\left|G^{f}(a, x)\right| \leqslant \frac{2^{a} C_{4}(a)}{(1-x)^{b-1}} \int_{x}^{1}(1-y)^{b-1} d y \leqslant C_{2}(a)(1-x)
\end{array}
$$


where we increased $C_{0}(a)$ and $C_{2}(a)$ if necessary. Thus the bounds (A.4) and (A.6) hold. Moreover, by (A.8) and (A.9),

$$
\left|x \frac{f^{\prime}(x)}{f(x)}\right| L^{f}(a, x)=|b-1| \frac{x}{1-x} L^{f}(a, x) \leqslant \begin{cases}C_{1}(a)(1+|\log x|) & \text { if } 0 \leqslant x<\frac{1}{2} \\ C_{1}(a) & \text { if } \frac{1}{2} \leqslant x<1\end{cases}
$$

proving the bound (A.5).

We now consider the case $f(x)=\left(\frac{x}{x+1}\right)^{b}$. Let $a \in D\left(M_{f}\right)=(-b, 0)$. Then

$$
\begin{aligned}
&\left|\psi_{0}^{f}(a)-\log y\right| y^{a-1} f(y) \leqslant \begin{cases}C_{4}(a)(1-\log y) y^{a+b-1} & \text { if } 0<y<1 \\
C_{4}(a)(1+\log y) y^{a-1} & \text { if } y \geqslant 1\end{cases} \\
&\left|\psi_{1}^{f}(a)+\psi_{0}^{f}(a) \log y-(\log y)^{2}\right| y^{a-1} f(y) \leqslant \begin{cases}C_{4}(a)\left(1+(\log y)^{2}\right) y^{a+b-1} & \text { if } 0<y<1 \\
C_{4}(a)\left(1+(\log y)^{2}\right) y^{a-1} & \text { if } y \geqslant 1 .\end{cases}
\end{aligned}
$$

Since $a+b>0,(3.8)$ and (A.7) give: for $0<x<1$,

$$
\begin{gathered}
L^{f}(a, x) \leqslant \frac{2^{b} C_{4}(a)}{x^{a+b}} \int_{0}^{x}(1-\log y) y^{a+b-1} d y \leqslant C_{0}(a)(1+|\log x|) \\
\left|G^{f}(a, x)\right| \leqslant \frac{2^{b} C_{4}(a)}{x^{a+b}} \int_{0}^{x}\left(1+(\log y)^{2}\right) y^{a+b-1} d y \leqslant C_{2}(a)\left(1+(\log x)^{2}\right) .
\end{gathered}
$$

Similarly, since $a<0$, the secondary expressions in (3.8) and (A.7) give: for $x \geqslant 1$,

$$
\begin{gathered}
L^{f}(a, x) \leqslant \frac{2^{b} C_{4}(a)}{x^{a}} \int_{x}^{\infty}(1+\log y) y^{a-1} d y \leqslant C_{0}(a)(1+|\log x|) \\
\left|G^{f}(a, x)\right| \leqslant \frac{2^{b} C_{4}(a)}{x^{a}} \int_{x}^{\infty}\left(1+(\log y)^{2}\right) y^{a-1} d y \leqslant C_{2}(a)\left(1+(\log x)^{2}\right)
\end{gathered}
$$

where we increased $C_{0}(a)$ and $C_{2}(a)$ if necessary. Thus the bounds (A.4) and (A.6) hold. Since $\left|x \frac{f^{\prime}(x)}{f(x)}\right|=b \frac{1}{x+1} \leqslant b$, (A.4) implies (A.5) completing the proof.

\section{Appendix Appendix B Lemmas used in Section 3 and Section 4}

Lemma B.1. Assume the polymer environment is such that $\log R^{1}, \log R^{2}, \log Y^{1}$, and $\log Y^{2}$ have finite second moments. Then $\mathbb{E}\left[\left(\log Z_{x}\right)^{2}\right]<\infty$ for any $x \in \mathbb{Z}_{+}^{2}$.

Proof: Since $\log Z_{k, 0}=\sum_{i=1}^{k} R_{i, 0}^{1}$ and $\log Z_{0, \ell}=\sum_{j=1}^{\ell} \log R_{0, j}^{2}, \log Z_{x}$ has finite second moment for each $x \in \mathbb{Z}_{+}^{2} \backslash \mathbb{N}^{2}$. If $x \in \mathbb{N}^{2}$, the recursion (2.1) implies that

$$
\begin{aligned}
\left(\log Y_{x}^{1}+\log Z_{x-\alpha_{1}}\right) & \wedge\left(\log Y_{x}^{2}+\log Z_{x-\alpha_{2}}\right) \\
\leqslant & \leqslant \frac{\log Z_{x}}{2} \leqslant\left(\log Y_{x}^{1}+\log Z_{x-\alpha_{1}}\right) \vee\left(\log Y_{x}^{2}+\log Z_{x-\alpha_{2}}\right) .
\end{aligned}
$$

Thus

$$
\left(\log Z_{x}\right)^{2} \leqslant 4\left(\log Y_{x}^{1}+\log Z_{x-\alpha_{1}}\right)^{2}+4\left(\log Y_{x}^{2}+\log Z_{x-\alpha_{2}}\right)^{2} .
$$

Since $\log Y^{1}$ and $\log Y^{2}$ have finite second moments, an inductive argument finishes the proof.

Lemma B.2. Suppose $f_{k}:(0, \infty) \rightarrow[0, \infty)$ for $k=1, \ldots, r$ and $a_{0}<a<a_{1}$ are real numbers such that $\left[a_{0}, a_{1}\right] \subset \bigcap_{k=1}^{r} D\left(M_{f_{k}}\right)$. Suppose we have a collection of independent random variables $\left\{X_{k}\right\}_{k=1}^{r}$ where $X_{k} \sim m_{f_{k}}(a)$ for all $1 \leqslant k \leqslant r$. Let $\mathbb{E}^{a}$ be the expectation corresponding to the product measure induced by $\left\{X_{k}\right\}_{k=1}^{r}$. 
Let $S=\sum_{k=1}^{r} \log X_{k}$ and $A: \mathbb{R}^{r} \rightarrow \mathbb{R}$ be a measurable function such that $\mathbb{E}^{a}\left[A\left(X_{1}, \ldots, X_{r}\right)^{2}\right]<\infty$ for all $a \in\left[a_{0}, a_{1}\right]$. Then

$$
\frac{\partial}{\partial a} \mathbb{E}^{a}\left[A\left(X_{1}, \ldots, X_{r}\right)\right]=\operatorname{Cov}^{a}\left(A\left(X_{1}, \ldots, X_{r}\right), S\right) \quad \text { for all } \quad a \in\left(a_{0}, a_{1}\right)
$$

and $\left(a_{0}, a_{1}\right) \ni a \mapsto \frac{\partial}{\partial a} \mathbb{E}^{a}\left[A\left(X_{1}, \ldots, X_{r}\right)\right]$ is continuous.

Proof: The joint density of $\left(\log X_{1}, \log X_{2}, \ldots, \log X_{r}\right)$ is given by

$$
g\left(x_{1}, \ldots, x_{r}\right)=\frac{e^{a \sum_{k=1}^{r} x_{k}}}{\prod_{k=1}^{r} M_{f_{k}}(a)} \prod_{k=1}^{r} f_{k}\left(e^{x_{k}}\right) .
$$

Thus the density of $S$ is given by

$$
h_{a}(s)=\frac{e^{a s}}{\prod_{k=1}^{r} M_{f_{k}}(a)} \int_{\mathbb{R}^{r-1}} f_{1}\left(e^{x_{1}}\right) f_{2}\left(e^{x_{2}-x_{1}}\right) \ldots f_{r}\left(e^{s-x_{r-1}}\right) d x_{1}, \ldots, x_{r-1}
$$

Therefore the joint density of $\left(\log X_{1}, \log X_{2}, \ldots, \log X_{r}\right)$ given that $S=s$ is

$$
\frac{g\left(x_{1}, \ldots, x_{r}\right) \mathbb{1}_{\left\{\sum_{k=1}^{r} x_{k}=s\right\}}}{h_{a}(s)}=\frac{\prod_{k=1}^{r} f_{k}\left(e^{x_{k}}\right) \mathbb{1}_{\left\{\sum_{k=1}^{r} x_{k}=s\right\}}}{\int_{\mathbb{R}^{r-1}} f_{1}\left(e^{x_{1}}\right) f_{2}\left(e^{x_{2}-x_{1}}\right) \ldots f_{r}\left(e^{s-x_{r-1}}\right) d x_{1}, \ldots, x_{r-1}},
$$

which has no $a$ dependence. Thus

$$
\begin{aligned}
\frac{\partial}{\partial a} \mathbb{E}^{a}\left[A\left(X_{1}, \ldots, X_{r}\right)\right] & =\frac{\partial}{\partial a} \int_{\mathbb{R}} \mathbb{E}^{a}\left[A\left(X_{1}, \ldots, X_{r}\right) \mid S=s\right] h_{a}(s) d s \\
& =\int_{\mathbb{R}} \mathbb{E}^{a}\left[A\left(X_{1}, \ldots, X_{r}\right) \mid S=s\right] \frac{\partial}{\partial a} h_{a}(s) d s \\
& =\int_{\mathbb{R}} \mathbb{E}^{a}\left[A\left(X_{1}, \ldots, X_{r}\right) \mid S=s\right] h_{a}(s)\left(s-\sum_{k=1}^{r} \frac{\partial}{\partial a} \log M_{f_{k}}(a)\right) d s \\
& =\operatorname{Cov}^{a}\left(A\left(X_{1}, \ldots, X_{r}\right), S\right) .
\end{aligned}
$$

The last equality comes from $\mathbb{E}[S]=\sum_{k=1}^{r} \mathbb{E}\left[\log X_{k}\right]=\sum_{k=1}^{r} \frac{\partial}{\partial a} \log M_{f_{k}}(a)$, by part (a) of Remark 3.2. The interchanging of the derivative and the integral is justified by the bound

$$
\int_{\mathbb{R}} \mathbb{E}\left[\left|A\left(X_{1}, \ldots, X_{r}\right)\right| \mid S=s\right] \sup _{a \in\left[a_{0}, a_{1}\right]}\left|\frac{\partial}{\partial a} h_{a}(s)\right| d s<\infty .
$$

Once we show that there is a constant $C$ depending only on $a_{0}$ and $a_{1}$ such that

$$
\sup _{a \in\left[a_{0}, a_{1}\right]}\left|\frac{\partial}{\partial a} h_{a}(s)\right| \leqslant C(1+|s|)\left(h_{a_{0}}(s)+h_{a_{1}}(s)\right)
$$

we will have the bound (B.2) since

$$
\begin{array}{r}
\int_{\mathbb{R}} \mathbb{E}\left[\left|A\left(X_{1}, \ldots, X_{r}\right)\right| \mid S=s\right](1+|s|) \\
h_{a_{j}}(s) d s=\mathbb{E}^{a_{j}}\left[\left|A\left(X_{1}, \ldots, X_{r}\right)\right|(1+|S|)\right] \\
\leqslant \mathbb{E}^{a_{j}}\left[A\left(X_{1}, \ldots, X_{r}\right)^{2}\right]^{\frac{1}{2}} \mathbb{E}^{a_{j}}\left[(1+|S|)^{2}\right]^{\frac{1}{2}} .
\end{array}
$$

The last expression is finite since $\mathbb{E}^{a_{j}}\left[A\left(X_{1}, \ldots, X_{r}\right)^{2}\right]<\infty$ by assumption, and $S$ is a finite sum of independent random variables each of which has finite exponential moments, by part (a) of Remark 3.2. Notice that the bound (B.2) also implies that $a \mapsto \frac{\partial}{\partial a} \mathbb{E}^{a}\left[A\left(X_{1}, \ldots, X_{r}\right)\right]$ is continuous. All that is left to do is verify the bound 
(B.3). To accomplish this, notice that equation (B.1) implies that $\frac{\partial}{\partial a} \log h_{a}(s)=$ $s-\mathbb{E}^{a}[S]$. So

$$
\sup _{a \in\left[a_{0}, a_{1}\right]}\left|\frac{\partial}{\partial a} h_{a}(s)\right| \leqslant C_{1}(1+|s|) \sup _{a \in\left[a_{0}, a_{1}\right]} h_{a}(s)
$$

where $C_{1}:=1 \vee \sup _{a \in\left[a_{0}, a_{1}\right]}\left|E^{a}[S]\right|$. Thus it suffices to show that $\sup _{a \in\left[a_{0}, a_{1}\right]} h_{a}(s)$ $\leqslant C_{2}\left(h_{a_{0}}(s)+h_{a_{1}}(s)\right)$ for some constant $C_{2}$ independent of $s$. By part (c) of Remark 3.2, $a \mapsto \mathbb{E}^{a}[S]$ is an increasing function. Therefore, for all $s \leqslant \mathbb{E}^{a_{0}}[S]$ the function $a \mapsto h_{a}(s)$ is non-increasing on $\left[a_{0}, a_{1}\right]$. Thus

$$
\sup _{a \in\left[a_{0}, a_{1}\right]} h_{a}(s) \leqslant h_{a_{0}}(s) \text { for all } s \leqslant \mathbb{E}^{a_{0}}[S]
$$

On the other hand, if $s>\mathbb{E}^{a_{0}}[S]$, then $\frac{\partial}{\partial a} \log \left(h_{a}(s) \exp \left(a\left(\mathbb{E}^{a_{1}}[S]-\mathbb{E}^{a_{0}}[S]\right)\right)\right)=$ $s-\mathbb{E}^{a}[S]+\mathbb{E}^{a_{1}}[S]-\mathbb{E}^{a_{0}}[S]>0$ for all $a \in\left[a_{0}, a_{1}\right]$. Thus for all $s>\mathbb{E}^{a_{0}}[S], a \mapsto$ $h_{a}(s) \exp \left(a\left(\mathbb{E}^{a_{1}}[S]-\mathbb{E}^{a_{0}}[S]\right)\right)$ is increasing on the interval $\left[a_{0}, a_{1}\right]$. Therefore,

$$
\sup _{a \in\left[a_{0}, a_{1}\right]} h_{a}(s) \leqslant C_{3} h_{a_{1}}(s) \text { for all } s>\mathbb{E}^{a_{0}}[S]
$$

where $C_{3}=\exp \left(\left(a_{1}-a_{0}\right)\left(\mathbb{E}^{a_{1}}[S]-\mathbb{E}^{a_{0}}[S]\right)\right)$. We now get the desired result with $C_{2}=1+C_{3}$.

Lemma B.3. Assume that the polymer environment is distributed as in (3.2) and let $\epsilon$ be small enough such that for all $|\lambda| \leqslant \epsilon, a_{1}+\lambda \in D\left(M_{f^{1}}\right)$ and $a_{2}-\lambda \in D\left(M_{f^{2}}\right)$. Let $(m, n) \in \mathbb{N}^{2}$ and $k \in \mathbb{N}$. Then, with notation as in $(3.5), Q_{m, n}^{\left(a_{1}+\lambda, a_{2}-\lambda\right)}\left(t_{1} \geqslant k\right)$ is stochastically non-decreasing in $\lambda$ and $Q_{m, n}^{\left(a_{1}+\lambda, a_{2}-\lambda\right)}\left(t_{2} \geqslant k\right)$ is stochastically nonincreasing in $\lambda$.

Proof:

$$
\frac{\partial}{\partial b_{i}} Q_{m, n}^{\left(b_{1}, b_{2}\right)}\left(t_{j} \geqslant k\right)=\frac{\partial}{\partial b_{i}}\left(\frac{1}{Z_{m, n}\left(b_{1}, b_{2}\right)} \sum_{x \in \Pi_{m, n}} \mathbb{1}_{\left\{t_{j} \geqslant k\right\}} W\left(b_{1}, b_{2}\right)\left(x_{\bullet}\right)\right) .
$$

If $i \neq j$, the sum in (B.4) has no $b_{i}$ dependence, so

$$
\frac{\partial}{\partial b_{i}} Q_{m, n}^{\left(b_{1}, b_{2}\right)}\left(t_{j} \geqslant k\right)=\frac{-1}{\left(Z_{m, n}\left(b_{1}, b_{2}\right)\right)^{2}}\left(\frac{\partial}{\partial b_{i}} Z_{m, n}\left(b_{1}, b_{2}\right)\right) \sum_{x, \in \Pi_{m, n}} \mathbb{1}_{\left\{t_{j} \geqslant k\right\}} W\left(b_{1}, b_{2}\right)\left(x_{\bullet}\right),
$$

which is non-positive by (3.18). If $i=j$, then by (3.17) and (3.18),

$$
\begin{aligned}
\frac{\partial}{\partial b_{i}} Q_{m, n}^{\left(b_{1}, b_{2}\right)}\left(t_{i} \geqslant k\right)= & \frac{\sum_{x, \in \Pi_{m, n}} \mathbb{1}_{\left\{t_{i} \geqslant k\right\}} \frac{\partial}{\partial b_{i}} W\left(b_{1}, b_{2}\right)(x .)}{Z_{m, n}\left(b_{1}, b_{2}\right)} \\
& -\left(\frac{\partial}{\partial b_{i}} \log Z_{m, n}\left(b_{1}, b_{2}\right)\right) \frac{\sum_{x \in \in \Pi_{m, n}} \mathbb{1}_{\left\{t_{i} \geqslant k\right\}} W\left(b_{1}, b_{2}\right)(x \cdot)}{Z_{m, n}\left(b_{1}, b_{2}\right)} \\
= & \operatorname{Cov}^{Q_{m, n}^{\left(b_{1}, b_{2}\right)}}\left(\sum_{k=1}^{t_{i}} L^{f^{i}}\left(b_{i}, H^{f^{i}}\left(b_{i}, \eta_{k}^{i}\right)\right), \mathbb{1}_{\left\{t_{i} \geqslant k\right\}}\right),
\end{aligned}
$$

which is non-negative. 


\section{Appendix Appendix C Properties of $\psi_{n}^{f}$}

\begin{tabular}{|l||c|c|}
\hline Model & $\psi_{n}^{f^{1}}\left(a_{1}\right)$ & $\psi_{n}^{f^{2}}\left(a_{2}\right)$ \\
\hline \hline IG & $(-1)^{n+1}\left(\Psi_{n}(\mu-\theta)-\delta_{n, 0} \log \beta\right)$ & $(-1)^{n+1}\left(\Psi_{n}(\theta)-\delta_{n, 0} \log \beta\right)$ \\
\hline G & $\Psi_{n}(\mu+\theta)-\delta_{n, 0} \log \beta$ & $(-1)^{n+1}\left(\Psi_{n}(\theta)-\Psi_{n}(\mu+\theta)\right)$ \\
\hline B & $\Psi_{n}(\mu+\theta)-\Psi_{n}(\mu+\theta+\beta)$ & $(-1)^{n+1}\left(\Psi_{n}(\theta)-\Psi_{n}(\mu+\theta)\right)$ \\
\hline IB & $(-1)^{n+1}\left(\Psi_{n}(\mu-\theta)-\Psi_{n}(\mu-\theta+\beta)\right.$ & $\Psi_{n}(\mu-\theta+\beta)+(-1)^{n+1} \Psi_{n}(\theta)$ \\
\hline
\end{tabular}

Figure C.9. $\psi_{n}^{f}$ functions for each of the four basic beta-gamma models.

By Abramowitz and Stegun (1964) (p.260 line 6.4.1) the polygamma function of order $n, \Psi_{n}(x)=\frac{\partial^{n+1}}{\partial x^{n+1}} \log \Gamma(x)$, has integral representation

$$
\Psi_{n}(x)=(-1)^{n+1} \int_{0}^{\infty} \frac{t^{n} e^{-x t}}{1-e^{-t}} d t
$$

Lemma C.1. For any $n \in \mathbb{N}$, the map $a \mapsto \frac{\Psi_{n+1}(a)}{\Psi_{n}(a)}$ is strictly increasing on $(0, \infty)$.

Proof: Fix $n \in \mathbb{N}$ and $a \in(0, \infty)$. We will show that $\frac{\partial^{2}}{\partial a^{2}} \log \left|\Psi_{n}(a)\right|>0$.

After substituting $y=e^{-t}$ in (C.1) we get

$$
\left|\Psi_{n}(a)\right|=\int_{0}^{\infty} y^{a-1} f(y) d y=M_{f}(a)
$$

where $f(y):=\frac{(-\log y)^{n}}{1-y} \mathbb{1}_{\{0<y<1\}}$. Note that $D\left(M_{f}\right)=(0, \infty)$. Now given a random variable $X \sim m_{f}(a)$, by part (c) of Remark 3.2,

$$
\frac{\partial^{2}}{\partial a^{2}} \log \left|\Psi_{n}(a)\right|=\frac{\partial^{2}}{\partial a^{2}} \log M_{f}(a)=\mathbb{V a r}[\log X]>0,
$$

since $X$ is non-degenerate.

Lemma C.2. Assume the polymer environment is distributed as in (3.2). Then

$$
\psi_{1}^{f^{1}}\left(a_{1}\right) \psi_{2}^{f^{2}}\left(a_{2}\right)+\psi_{1}^{f^{2}}\left(a_{2}\right) \psi_{2}^{f^{1}}\left(a_{1}\right)>0
$$

Proof: Recall that $\psi_{1}^{f^{j}}$ are always positive and by (C.1) $\Psi_{n}$ has sign $(-1)^{n+1}$ throughout $(0, \infty)$.

For the inverse-gamma model (1.2) with fixed constants $\beta>0$ and $\mu>\theta>0$, Table C.9 implies that $\psi_{2}^{f^{j}}\left(a_{j}\right)>0$ for $j=1,2$. The conclusion follows immediately.

For the gamma model (1.3) with fixed positive constants $\beta, \mu$, and $\theta$, by Table C.9

$$
\psi_{1}^{f^{1}}\left(a_{1}\right) \psi_{2}^{f^{2}}\left(a_{2}\right)+\psi_{1}^{f^{2}}\left(a_{2}\right) \psi_{2}^{f^{1}}\left(a_{1}\right)=-\Psi_{1}(\theta+\mu) \Psi_{2}(\theta)+\Psi_{1}(\theta) \Psi_{2}(\theta+\mu) .
$$

The quantity on the right hand side is positive if and only if

$$
\frac{\Psi_{2}(\theta+\mu)}{\Psi_{1}(\theta+\mu)}>\frac{\Psi_{2}(\theta)}{\Psi_{1}(\theta)}
$$

which holds true by Lemma C.1 with $n=1$. 
For the beta model (1.4) with fixed positive constants $\beta$, $\mu$, and $\theta$, using Table C.9

$$
\begin{aligned}
& \psi_{1}^{f^{1}}\left(a_{1}\right) \psi_{2}^{f^{2}}\left(a_{2}\right)+\psi_{1}^{f^{2}}\left(a_{2}\right) \psi_{2}^{f^{1}}\left(a_{1}\right)>0 \quad \Leftrightarrow \\
& \frac{\psi_{2}^{f^{1}}\left(a_{1}\right)}{\psi_{1}^{f^{1}}\left(a_{1}\right)}>-\frac{\psi_{2}^{f^{2}}\left(a_{2}\right)}{\psi_{1}^{f^{2}}\left(a_{2}\right)} \\
& \frac{\Psi_{2}(\theta+\mu+\beta)-\Psi_{2}(\theta+\mu)}{\Psi_{1}(\theta+\mu+\beta)-\Psi_{1}(\theta+\mu)}>\frac{\Psi_{2}(\theta+\mu)-\Psi_{2}(\theta)}{\Psi_{1}(\theta+\mu)-\Psi_{1}(\theta)} .
\end{aligned}
$$

By Cauchy's mean value theorem there exist constants $\theta<\xi_{1}<\theta+\mu<\xi_{2}<$ $\theta+\mu+\beta$ such that the left and right-hand sides of (C.2) equal $\frac{\Psi_{3}\left(\xi_{2}\right)}{\Psi_{2}\left(\xi_{2}\right)}$ and $\frac{\Psi_{3}\left(\xi_{1}\right)}{\Psi_{2}\left(\xi_{1}\right)}$ respectively. Lemma C.1 with $n=2$ now gives (C.2).

For the inverse-beta model (1.5) with fixed constants $\beta>0$ and $\mu>\theta>0$, by Table C.9, $\psi_{2}^{f^{1}}\left(a_{1}\right)>0, \psi_{1}^{f^{2}}\left(a_{2}\right)>\Psi_{1}(-\theta+\mu+\beta)$, and $\psi_{2}^{f^{2}}\left(a_{2}\right)>\Psi_{2}(-\theta+\mu+\beta)$. Therefore

$$
\begin{aligned}
\psi_{1}^{f^{1}}\left(a_{1}\right) \psi_{2}^{f^{2}}\left(a_{2}\right)+ & \psi_{1}^{f^{2}}\left(a_{2}\right) \psi_{2}^{f^{1}}\left(a_{1}\right) \\
& >\psi_{1}^{f^{1}}\left(a_{1}\right) \Psi_{2}(-\theta+\mu+\beta)+\Psi_{1}(-\theta+\mu+\beta) \psi_{2}^{f^{1}}\left(a_{1}\right) \\
& =\Psi_{1}(-\theta+\mu) \Psi_{2}(-\theta+\mu+\beta)-\Psi_{1}(-\theta+\mu+\beta) \Psi_{2}(-\theta+\mu)
\end{aligned}
$$

Letting $x=-\theta+\mu$, the last line is positive if and only if

$$
\frac{\Psi_{2}(x+\beta)}{\Psi_{1}(x+\beta)}>\frac{\Psi_{2}(x)}{\Psi_{1}(x)}
$$

which holds true by Lemma C.1 with $n=1$.

\section{References}

M. Abramowitz and I. A. Stegun. Handbook of mathematical functions with formulas, graphs, and mathematical tables, volume 55 of National Bureau of Standards Applied Mathematics Series. For sale by the Superintendent of Documents, U.S. Government Printing Office, Washington, D.C. (1964). MR0167642.

G. Amir, I. Corwin and J. Quastel. Probability distribution of the free energy of the continuum directed random polymer in $1+1$ dimensions. Comm. Pure Appl. Math. 64 (4), 466-537 (2011). MR2796514.

M. Balázs, E. Cator and T. Seppäläinen. Cube root fluctuations for the corner growth model associated to the exclusion process. Electron. J. Probab. 11, no. 42, 1094-1132 (2006). MR2268539.

M. Balázs, J. Quastel and T. Seppäläinen. Fluctuation exponent of the KPZ/stochastic Burgers equation. J. Amer. Math. Soc. 24 (3), 683-708 (2011). MR2784327.

M. Balázs, F. Rassoul-Agha and T. Seppäläinen. Large deviations and wandering exponent for random walk in a dynamic beta environment. ArXiv Mathematics e-prints (2018). arXiv: 1801.08070.

G. Barraquand and I. Corwin. Random-walk in beta-distributed random environment. Probab. Theory Related Fields 167 (3-4), 1057-1116 (2017). MR3627433.

A. Borodin and I. Corwin. Macdonald processes. Probab. Theory Related Fields 158 (1-2), 225-400 (2014). MR3152785. 
A. Borodin, I. Corwin and P. Ferrari. Free energy fluctuations for directed polymers in random media in $1+1$ dimension. Comm. Pure Appl. Math. 67 (7), 1129-1214 (2014). MR3207195.

A. Borodin, I. Corwin and D. Remenik. Log-gamma polymer free energy fluctuations via a Fredholm determinant identity. Comm. Math. Phys. 324 (1), 215-232 (2013). MR3116323.

E. Cator and P. Groeneboom. Second class particles and cube root asymptotics for Hammersley's process. Ann. Probab. 34 (4), 1273-1295 (2006). MR2257647.

H. Chaumont and C. Noack. Characterizing stationary 1+1 dimensional lattice polymer models. ArXiv Mathematics e-prints (2017). arXiv: 1708.07187.

F. Comets. Directed polymers in random environments, volume 2175 of Lecture Notes in Mathematics. Springer, Cham (2017). ISBN 978-3-319-50486-5; 9783-319-50487-2. Lecture notes from the 46th Probability Summer School held in Saint-Flour, 2016.

I. Corwin, T. Seppäläinen and H. Shen. The strict-weak lattice polymer. J. Stat. Phys. 160 (4), 1027-1053 (2015). MR3373650.

D. Huse and C. Henley. Pinning and roughening of domain walls in Ising systems due to random impurities. Phys. Rev. Lett. 54 (1985). DOI: 10.1103/PhysRevLett.54.2708.

J. Z. Imbrie and T. Spencer. Diffusion of directed polymers in a random environment. J. Statist. Phys. 52 (3-4), 609-626 (1988). MR968950.

G. R. Moreno Flores, T. Seppäläinen and B. Valkó. Fluctuation exponents for directed polymers in the intermediate disorder regime. Electron. J. Probab. 19, no. 89, 28 (2014). MR3263646.

N. O'Connell and J. Ortmann. Tracy-Widom asymptotics for a random polymer model with gamma-distributed weights. Electron. J. Probab. 20, no. 25, 18 (2015). MR3325095.

N. O'Connell and M. Yor. Brownian analogues of Burke's theorem. Stochastic Process. Appl. 96 (2), 285-304 (2001). MR1865759.

T. Seppäläinen. Scaling for a one-dimensional directed polymer with boundary conditions. Ann. Probab. 40 (1), 19-73 (2012). MR2917766.

T. Seppäläinen and B. Valkó. Bounds for scaling exponents for a $1+1$ dimensional directed polymer in a Brownian environment. ALEA Lat. Am. J. Probab. Math. Stat. 7, 451-476 (2010). MR2741194.

T. Thiery. Stationary measures for two dual families of finite and zero temperature models of directed polymers on the square lattice. J. Stat. Phys. 165 (1), 44-85 (2016). MR3547834.

T. Thiery and P. Le Doussal. On integrable directed polymer models on the square lattice. J. Phys. A 48 (46), 465001, 41 (2015). MR3418005. 